\title{
Does the Holton-Tan Mechanism Explain How the Quasi-Biennial Oscillation Modulates the Arctic Polar Vortex?
}

\author{
CHAIM I. GARFINKEL \\ Department of Earth and Planetary Science, Johns Hopkins University, Baltimore, Maryland \\ TIFFANY A. SHAW \\ Department of Earth and Environmental Sciences, and Department of Applied Physics and Applied \\ Mathematics, Columbia University, New York, New York \\ DENNIS L. HARTMANN \\ Department of Atmospheric Science, University of Washington, Seattle, Washington \\ DARRYN W. WAUGH \\ Department of Earth and Planetary Science, Johns Hopkins University, Baltimore, Maryland
}

(Manuscript received 10 August 2011, in final form 3 December 2011)

\begin{abstract}
Idealized experiments with the Whole Atmosphere Community Climate Model (WACCM) are used to explore the mechanism(s) whereby the stratospheric quasi-biennial oscillation (QBO) modulates the Northern Hemisphere wintertime stratospheric polar vortex. Overall, the effect of the critical line emphasized in the Holton-Tan mechanism is less important than the effect of the mean meridional circulation associated with QBO winds for the polar response to the QBO. More specifically, the introduction of easterly winds at the equator near $50 \mathrm{hPa}$ 1) causes enhanced synoptic-scale Eliassen-Palm flux (EPF) convergence in the subtropics from 150 to $50 \mathrm{hPa}$, which leads to the subtropical critical line moving poleward in the lower stratosphere, and 2) creates a barrier to planetary wave propagation from subpolar latitudes to midlatitudes in the middle and upper stratosphere (e.g., less equatorward EPF near $50^{\circ} \mathrm{N}$ ), which leads to enhanced planetary wave convergence in the polar vortex region. These two effects are mechanistically distinct; while the former is related to the subtropical critical line, the latter is due to the mean meridional circulation of the QBO. All of these effects are consistent with linear theory, although the evolution of the entire wind distribution is only quasi-linear because induced zonal wind changes cause the wave driving to shift and thereby positively feed back on the zonal wind changes. Finally, downward propagation of the QBO in the equatorial stratosphere, upper stratospheric equatorial zonal wind, and changes in the tropospheric circulation appear to be less important than lower stratospheric easterlies for the polar stratospheric response. Overall, an easterly QBO wind anomaly in the lower stratosphere leads to a weakened stratospheric polar vortex, in agreement with previous studies, although not because of changes in the subtropical critical line.
\end{abstract}

\section{Introduction}

The strength of the Northern Hemisphere stratospheric wintertime polar vortex is highly variable. Although much of this variability is stochastic (Holton and Mass 1976),

Corresponding author address: Chaim I. Garfinkel, Department of Earth and Planetary Science, Johns Hopkins University, Baltimore, MD 21218.

E-mail: cig4@jhu.edu
Garfinkel et al. (2010) found that approximately $40 \%$ of the variability on interseasonal time scales may be linked to variability occurring outside of the polar stratosphere. One of the main sources of predictable external variability is the quasi-biennial oscillation (QBO). Arctic polar cap temperatures are significantly colder, and polar stratospheric heights significantly lower, when lower stratospheric QBO winds (e.g., winds near $50 \mathrm{hPa}$ ) are westerly than when they are easterly. Both modelingbased studies (e.g., Hampson and Haynes 2006; Pascoe 
et al. 2006; Naito and Yoden 2006; Kinnersley and Tung 1999; Naoe and Shibata 2010; Calvo et al. 2007; O'Sullivan and Young 1992, hereafter OY92; Niwano and Takahashi 1998) and reanalysis-based studies (e.g., $\mathrm{Hu}$ and Tung 2002; Hitchman and Huesmann 2009; Ruzmaikin et al. 2005; Garfinkel and Hartmann 2007) have shown a robust effect of the QBO in the polar stratosphere. This effect is referred to as the Holton-Tan (HT) effect in the rest of this paper. The mechanism(s) behind this effect are less clear, however.

Holton and Tan (1980) were the first to propose a mechanism of how the QBO might influence the vortex. They hypothesized that the QBO modulates the location of the subtropical critical wind line, thereby affecting the propagation of planetary waves in the stratosphere. In the rest of this paper, we refer to the mechanism presented in Holton and Tan (1980) involving the subtropical critical line as the HT mechanism. Holton and Tan (1980) were unable to find any clear differences in the Eliassen-Palm flux (EPF) between the easterly and westerly phases of the QBO, however. Although more recent studies do find a robust change in EPF due to the QBO (Garfinkel and Hartmann 2008; Yamashita et al. 2011), a change in EPF due to the QBO does not prove the HT mechanism. In particular, the proximate cause whereby the QBO influences planetary wave propagation need not be the subtropical critical line (e.g., this paper will show that the QBO's meridional circulation influences planetary wave propagation). In addition, shear in QBO wind profiles complicates application of the HT mechanism. Because the characteristic vertical wavelength of the shear $(\sim 20 \mathrm{~km})$ is of the same order of magnitude as the wavelength of vertically propagating planetary Rossby waves (Matsuno 1970), it is difficult to predict how exactly Rossby wave propagation will be changed when the direction of the critical line shift depends on vertical level.

Furthermore, it is difficult to isolate the mechanism(s) through which the QBO influences the vortex in observations and in GCMs, because they contain unrelated variability, which can also influence the vortex. For example, variability in sea surface temperatures (SSTs) can influence the tropospheric planetary wave pattern (Garfinkel and Hartmann 2008; Fletcher and Kushner 2011; Hurwitz et al. 2011) and, because the HT effect is sensitive to the magnitude of the planetary wave forcing from the troposphere (Holton and Austin 1991; O'Sullivan and Dunkerton 1994), the HT mechanism can be masked by unrelated tropospheric variability (Wei et al. 2007; Garfinkel and Hartmann 2007; Calvo et al. 2009). Naoe and Shibata (2010) and Yamashita et al. (2011) investigated the HT mechanism in a comprehensive GCM, and although the polar vortex is modulated as expected, wave propagation in the midlatitudes (i.e., near $50 \mathrm{hPa}, 40^{\circ} \mathrm{N}$ ) does not follow the HT mechanism. Finally, Pascoe et al. (2006) and Gray et al. $(2001,2004)$ found that the polar vortex is influenced by winds in the equatorial upper stratosphere; this effect is likely not explainable by the HT mechanism.

Simplified modeling studies are thus essential for a complete understanding of the mechanisms whereby the QBO influences the vortex. ${ }^{1}$ However, modeling studies of the HT mechanism have produced mixed results. Holton and Austin (1991) found that a sheared QBO influences polar vortex evolution over a narrow range of planetary wave forcings in a primitive equation model. But they also found little change in EPF propagation and convergence poleward of the subtropical critical line. Naito and Yoden (2006) found that EPF convergence in the flanks of the QBO region and in the polar vortex region is changed by QBO wind anomalies in a simplified dry GCM, although changes near $40^{\circ} \mathrm{N}, 50 \mathrm{hPa}$ are less clear. The proximate cause whereby lower stratospheric easterly QBO (EQBO) winds influence subpolar latitudes and weaken the vortex is not clearly explained in any of the previously mentioned studies.

OY92 studied the sensitivity of the time-mean winter state to a nonsheared equatorial zonal wind profile. Anomalous QBO winds influence tropical-extratropical coupling by altering the propagation of Rossby wave activity. For realistic tropospheric wave amplitude, a nonlinear critical layer develops north of the tropical easterlies, which affects planetary waves in the polar region. $^{2}$ Linear theory appears incapable of describing the polar response in the model used by OY92. On the other hand, Hauck and Wirth (2001) found that a linear quasigeostrophic (QG) model on the sphere can explain the effect of the QBO in the polar region. Neither of these studies [nor Holton and Austin (1991) nor Naito and Yoden (2006)] uses models that realistically resolve tropospheric dynamics (i.e., stationary planetary waves), and it is conceivable that the effect of the QBO in the troposphere (cf. Garfinkel and Hartmann 2011b, hereafter GH11b) may increase tropospheric planetary wave driving of the stratosphere. The precise mechanism(s) of how the QBO influences the polar vortex, and the relevance of linear theory for the observed effect, remain unclear.

To better understand the mechanism(s), we analyze a series of perpetual January and February Whole Atmosphere Community Climate Model (WACCM) runs

\footnotetext{
${ }^{1}$ Holton and Tan (1980) themselves note that "identification of the physical mechanisms involved will probably require careful studies with numerical models."

${ }^{2}$ Wave propagation and convergence is influenced by critical lines up to $20^{\circ}$ latitude away in the troposphere as well (Randel and Held 1991).
} 

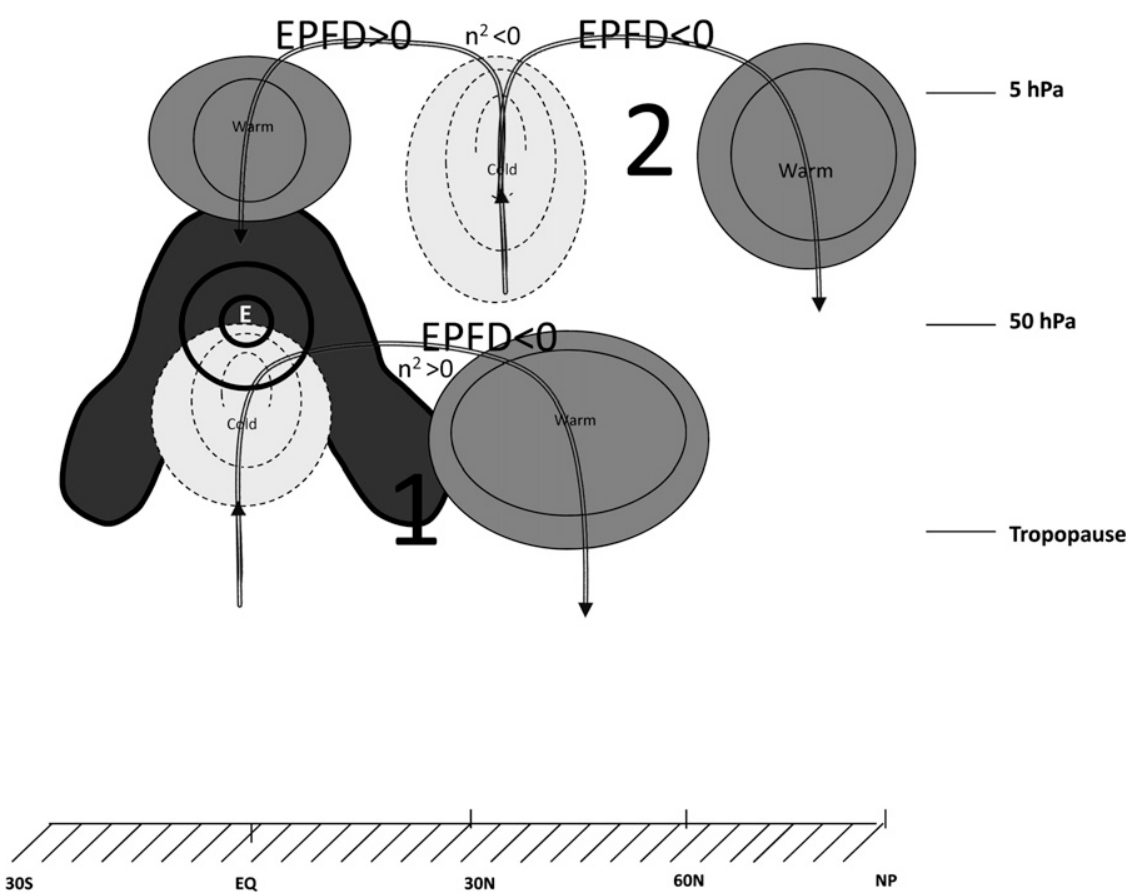

FIG. 1. Schematic of the response of the extratropics to the QBO 16-30 days after branching. Arrows denote the mass-weighted circulation. Thin contours are for temperature. All features, except for the easterly maxima at the equator (denoted with a thick contour), are a response to the EQBO winds rather than being due directly to the externally imposed torque. Changes in EPFD are directly related to changes in the index of refraction. In region 1 (subtropical lower stratosphere), the axisymmetric meridional circulation of the QBO in thermal wind balance dominates (see GH11b), although the poleward boundary of the easterlies and the latitude of the subsidence is modulated by eddies. That is, eddies propagating from the troposphere are influenced by the subtropical critical line and break in the lower stratosphere throughout the midlatitudes, forcing a TEM circulation that warms the midlatitude lower stratosphere. In region 2 (midlatitude upper stratosphere), subpolar Rossby waves are restricted from propagating into the subtropics because of a decrease in midlatitude index of refraction and therefore break closer to the pole. These Rossby waves lead to a TEM circulation that warms the pole. The warm anomaly reaches the lower stratosphere in the third and fourth month after branching. The effects in regions 1 and 2 are mechanistically distinct.

with an imposed QBO. WACCM runs with a neutral QBO stratospheric wind profile are compared to runs with an EQBO stratospheric wind profile. The SSTs and radiative forcing, as well as every other model parameterization except for the QBO, are fixed in all runs presented. In addition, the vertical shear of the QBO can be carefully controlled, thus enabling us to understand the role that shear may play in Rossby wave propagation. In particular, we will compare the response to EQBO profiles that are identical in the lower stratosphere but change in the upper stratosphere from a neutral QBO profile to westerlies as in observed QBO profiles. The model used here is more realistic than those in OY92 and Naito and Yoden (2006) because it includes a realistic dynamically active troposphere, but it does not contain any unrelated external variability. It is therefore an appropriate test bed for understanding how the QBO influences the polar vortex.
We will show that linear theory explains how lower stratospheric QBO anomalies influence Rossby wave propagation and weaken the vortex. Briefly, EQBO winds change the QG index of refraction in the lower stratosphere near $20^{\circ}-30^{\circ} \mathrm{N}$ and in the midstratosphere near $40^{\circ}-50^{\circ} \mathrm{N}$. These two effects are mechanistically distinct, and only the former is expected from the HT mechanism. These changes influence EPF propagation and lead to a residual circulation that warms the midlatitude lower stratosphere and the polar vortex. See Fig. 1 for a schematic.

After introducing the diagnostic tools and model runs used (section 2), we will show that WACCM generates a realistic HT effect in response to a downward propagating QBO (section 3). We will then show that this response is captured by a model run where the QBO phase is held fixed (i.e., the QBO profile does not propagate 


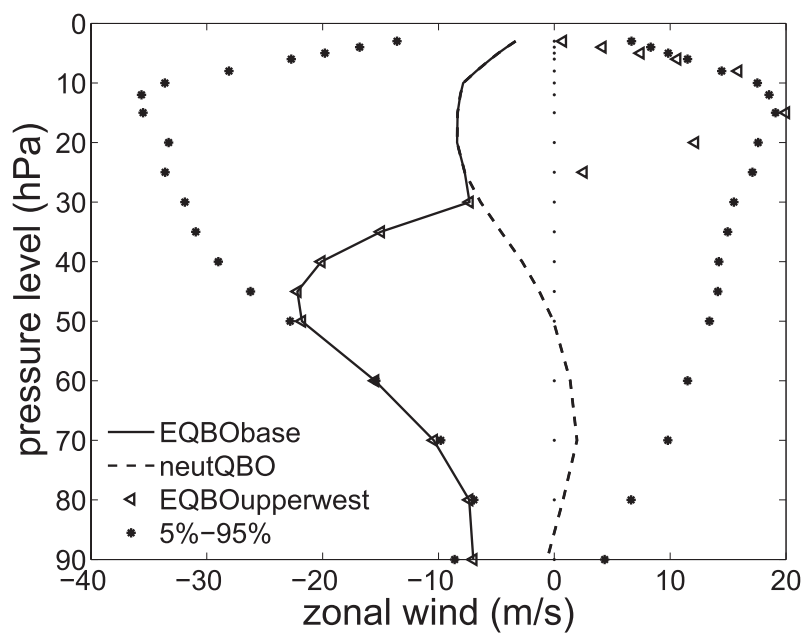

FIG. 2. The QBO profiles relaxed toward in the WACCM runs. Stars indicate the $5 \%-95 \%$ range of variability of equatorial zonal wind from May 1953 to April 2007.

downward) and also in a model run without upper stratospheric and midstratospheric QBO wind anomalies. Section 4 will show that the QBO does not affect the polar vortex through its influence on the troposphere. Rather, section 5 will show that linear QG theory explains how the QBO influences stratospheric wave propagation and the vortex. Section 6 will show that linear QG theory explains the polar response to realistic mid- and upper stratospheric equatorial winds as well. We expect that linear QG theory explains the influence of the QBO on the polar vortex in observations and in more complicated GCMs as well.

\section{Model runs and methodology}

\section{a. WACCM simulations}

WACCM version 3.1.9 (Marsh et al. 2007; Garcia et al. 2007; Richter et al. 2008) is run with fixed SSTs, land surface and ice, and perpetual 15 January or 5 February radiative forcing. Interactive chemistry is turned off. QBO wind anomalies are maintained through relaxation to a specified zonal mean zonal wind (Matthes et al. 2010; GH11b). See GH11b for more details of the model runs and for a comparison of the model's mean state to reanalysis data.

Two types of EQBO runs are explored. The first are long quasi-steady equilibrium runs in which the EQBO relaxation is always present (see Table 1 ). Three different EQBO profiles are used to explore sensitivity of the HT effect to the details of the QBO profile. Figure 2 shows the QBO wind profiles toward which the model is relaxed. Details of the profiles will be presented in section 3 . In the second type we (i) branch off the instantaneous
TABLE 1. Different WACCM runs for understanding the effect of the QBO on the vortex. For the perpetual February $3 \times$ EQBO branch run, the first number of "run length" denotes the number of ensemble members, each of which extends for 4 months. For all other runs, the run length denotes the number of months retained after the first 10 months are discarded. "Month" indicates whether radiative conditions are held fixed to 15 Jan or 5 Feb. GH11b (Fig. 3 and surrounding discussion) compares the mean state of the neutQBO run to the mean state in the reanalysis data.

\begin{tabular}{lcc}
\hline \multicolumn{3}{c}{ Different runs for QBO influence on vortex } \\
\hline \multicolumn{1}{c}{ QBO profile } & Month & Run length \\
\hline neutQBO & Jan & 463 \\
neutQBO & Feb & 225 \\
$3 \times$ EQBO & Feb & $68 \times 4$ \\
QBOprop & Jan & 558 \\
EQBOupperwest & Jan & 278 \\
EQBOupperwest & Feb & 181 \\
EQBObase & Jan & 168 \\
EQBObase & Feb & 167 \\
$3 \times$ EQBO & Jan & 219 \\
$3 \times$ EQBO & Feb & 182 \\
\hline
\end{tabular}

atmospheric state at the beginning of each month of a neutral QBO control run (hereafter neutQBO), (ii) relax the model toward an EQBO wind profile, and then (iii) integrate each ensemble member for an additional 120 days. A total of 68 ensemble members is created. We thus generate a large ensemble of the transient response to EQBO winds. The methodology is somewhat similar to that in OY92, although we test the response to QBO winds in a large ensemble of switch-on runs as opposed to a switch-on run from a single representative state. To improve the signal-to-noise ratio, QBO wind anomalies 3 times stronger than is realistic are used (hereafter $3 \times$ EQBO). The atmosphere can smoothly adjust to the EQBO equatorial stratospheric profile because the relaxation time scale for the nudging is no faster than 10 days. Finally, we also analyze a perpetual January WACCM run not discussed in GH11b where the winds are relaxed to downward-propagating observed QBO winds from September 1957 to December 2004 (QBOprop in Table 1). Section 3 will show that the HT effect is similar in all types of QBO runs, while sections 4 and 5 will focus on the mechanism in the ensemble of branch runs where the diagnostics are cleanest and easiest to understand.

The version of WACCM used here has too little stratospheric polar vortex variability and too few major sudden stratospheric warmings (SSWs; Richter et al. 2008); therefore, we cannot analyze the effect of the QBO on major SSW frequency. However, the HT mechanism should operate even in the absence of SSWs. In fact, an SSW can mask changes in Rossby wave propagation due to the QBO. In particular, an SSW will lower the polar zero wind line to the lower stratosphere, thereby causing 
large changes in Rossby wave propagation throughout the stratosphere. These changes in Rossby wave propagation can mask any extratropical anomaly induced by the QBO [unless large composites are created as in Naito and Yoden (2006)]. Even though model configurations with a more realistic SSW frequency are necessary to confirm results shown here, we expect that the WACCM runs realistically capture the mechanism by which the QBO influences the vortex. If anything, the modeled response to the $\mathrm{QBO}$ in the polar region might be amplified in a model with a more active stratosphere.

The QBO influences the vortex in our perpetual February and January runs even though the strongest observational HT effect is in early winter. The origin for the seasonality of the HT effect is, as yet, unclear. In fact, some models simulate a stronger HT effect later in winter (e.g., Niwano and Takahashi 1998; Naoe and Shibata 2010), opposite to the seasonality of the conventional HT effect. Our methodology precludes (convincing) conclusions regarding seasonality of the observed HT effect. Nevertheless, we expect that the mechanism presented for the effect of the QBO is relevant to early winter as well, as variability of the early winter vortex (before SSW events) is more linear and hence more amenable to arguments based on linear theory (e.g., Hu and Tung 2002).

\section{b. Statistical significance}

The methodology for computing statistical significance is different between the ensemble of branch runs and the quasi-steady equilibrium runs. For the quasiequilibrium runs, statistical significance is determined by a two-tailed Student's $t$ difference of means test. Because we compare runs with fixed external forcings, the number of degrees of freedom (DOFs) used for significance testing is less than the number of days of data available. To account for this, we count two consecutive months as one DOF. Similar results are obtained in the polar vortex region if we compute the DOFs following Bretherton et al. [1999, Eq. (31)].

For the $3 \times \mathrm{EQBO}$ branch runs, we use the following Monte Carlo test to estimate the relative likelihood of an anomaly appearing by chance. The neutQBO run is divided up into 15-day intervals. Sixty-eight unique intervals (corresponding to the 68 ensemble members) are chosen at random from the neutQBO run. The difference between these 68 intervals and the following interval (as well as the intervals $16-30$ days later, $31-45$ days later, and 46-60 days later) is computed. This procedure is repeated 1000 times, thus generating a probability distribution function (PDF). The observed difference after branching with EQBO winds is compared to the probability distribution function generated by the Monte Carlo procedure. A similar test is performed for differences four months apart for stationary wave EPF in the fourth month after branching. In the results presented below, probabilities exceeding the $2.5 \%$ or $97.5 \%$ level by this Monte Carlo test are indicated by shading. Nevertheless, all anomalies are forced by the QBO because we are comparing matched pairs of simulations (e.g., OY92). Therefore, even anomalies not marked as statistically significant by the Monte Carlo test are a direct response to the QBO.

\section{c. Diagnostic tools}

Three diagnostic tools are used to understand how Rossby wave propagation changes in the presence of EQBO winds: EPF cross sections, a linear spherical QG model, and the QG index of refraction. EPF convergence diagnoses the influence of finite-amplitude waves on the mean flow, and vectors approximately diagnose the propagation of Rossby waves. Cross sections of the EPF are therefore the clearest diagnostic of how the QBO influences Rossby waves in our WACCM runs. EPF is calculated as in Garfinkel and Hartmann (2008), except that here we use the QG EPF. Results are qualitatively similar in the extratropics if we use the full EPF, although the QG EPF underestimates the effect of the QBO by up to $50 \%$ equatorward of $10^{\circ}$ (not shown).

To understand how the QBO influences EPF, we examine the index of refraction $n^{2}$ for Rossby waves. Rossby waves are expected to refract toward regions with a higher index of refraction and converge at critical lines where $n^{2} \rightarrow \infty$ (Matsuno 1970). Finally, we also use the linear spherical QG model of Harnik and Lindzen (2001) to analyze changes in Rossby wave propagation for a prescribed QBO-dependent zonal mean wind and temperature basic state. The model calculates a steadystate wave solution to a specified lower boundary zonal wavenumber perturbation. The steady-state wave solution to a tropospheric Rossby wave source for a neutral QBO background state is compared to a similar solution but for an EQBO background state. Differences in stratospheric wave propagation are a response to the altered QBO-related boundary conditions only. The steady wave solution can then be used to derive the meridional and vertical wavenumbers according to Eqs. (12) and (13) of Harnik and Lindzen (2001). The two wavenumbers describe the vertical and meridional propagation separately and can be directly related to the index of refraction. ${ }^{3}$ Vertical (meridional) wave propagation is

\footnotetext{
${ }^{3}$ The $\operatorname{Re}\left(\psi_{z z} / \psi\right)=-m^{2}$ and $\operatorname{Re}\left(\psi_{y y} / \psi\right)=-l^{2} \cdot n^{2} / N^{2}=l^{2}+$ $f^{2} a^{2} m^{2} / N^{2}$ in spherical coordinates, where $a$ is the earth's radius, $m$ is the vertical wavenumber, $l$ is the horizontal wavenumber, $f$ is the Coriolis parameter, $N$ the buoyancy frequency, and $\psi$ is the streamfunction in the QG model.
} 
governed by $m^{2}\left(l^{2}\right)$. In particular, waves reflect in the vertical (meridional) where $m^{2}<0\left(l^{2}<0\right)$ and converge where $m^{2} \rightarrow \infty\left(l^{2} \rightarrow \infty\right)$, similar to the index of refraction. The vertical reflecting surface occurs where $m^{2}=0$; likewise, the meridional reflecting surface or meridional waveguide (MWG) occurs where $l^{2}=0$. See Harnik and Lindzen (2001), Harnik (2002), and Shaw et al. (2010) for more details. By comparing the index of refraction, the wave propagation in the QG model, and the observed EPF diagnostics in WACCM, we diagnose how wave propagation is modulated by the QBO. Because the HT mechanism hypothesizes that EPF convergence and critical lines for Rossby waves will be modulated by the QBO, these three diagnostic tools should capture the HT mechanism. These diagnostics will show that the HT mechanism explains the effect of the QBO in the subtropics, but that aspects of linear theory unrelated to the subtropical critical line are important in the polar region.

\section{Extratropical effects of the QBO}

In this section, we will show that the effect of the QBO in the polar and subtropical lower stratosphere is similar among all experiments performed. We then will discuss, in sections 4 and 5, the underlying mechanism in the most idealized experiment (i.e., the ensemble of branch runs) where the dynamics are cleanest and easiest to understand. We begin with the most realistic experiment considered in this paper: the response to four different QBO phases of a downward propagating QBO (QBOprop in Table 1).

Figure 3 shows the difference in Pacific sector (i.e., $130^{\circ}-210^{\circ} \mathrm{E}$; results insensitive to this definition) zonal wind and zonally averaged temperature between months with anomalous easterlies and westerlies at 10,20,40, and $70 \mathrm{hPa}$. The characteristic meridional circulation of the QBO in the tropics and subtropics is evident in all plots, as discussed in Plumb (1982), Plumb and Bell (1982), Garfinkel and Hartmann (2011a, hereafter GH11a), and GH11b. EQBO winds in the lower stratosphere require a cold anomaly at the equator below the peak tropical easterlies to maintain thermal wind balance. Subtropical zonal wind and temperature anomalies of the opposite sign to the tropical anomalies are also present. A zonal wind anomaly arches down to the tropopause near $15^{\circ}-$ $20^{\circ} \mathrm{N}$ to maintain thermal wind balance. The precise boundary between easterlies and westerlies and between subsidence and upwelling is modulated by wave convergence, to be discussed in section 5. For a sheared QBO, this circulation is repeated, but with opposite sign, higher in the stratosphere. All of the significant tropical and subtropical anomalies in Fig. 3 are consistent with this meridional circulation of the QBO. The polar temperature anomalies are not part of this meridional circulation, however, and a thorough understanding of how these polar temperature anomalies develop is our main goal.

When easterlies peak in the lower stratosphere (i.e., Figs. 3g,h), anomalies of the lower stratospheric temperature are strongest and are statistically significant. In the rest of this paper, we will focus on the QBO phase shown in Figs. 3g and $3 \mathrm{~h}$ where we find strong tropical lower stratospheric easterlies, upper stratospheric westerlies, and a weakened vortex.

Figure 4 shows zonally averaged cross sections of temperature anomalies in the quasi-steady equilibrium runs. Figures $4 \mathrm{a}$ and $4 \mathrm{~b}$ show the response to EQBO wind anomalies whose phase is similar to that in Figs. $3 \mathrm{~g}$ and $3 \mathrm{~h}$ (cf. triangles in Fig. 2). The polar response to a sheared QBO profile with easterlies in the lower stratosphere does not depend on the downward propagation of the QBO. The magnitude of the response differs between Figs. 3 and 4. This difference likely arises because we subtract an EQBO composite from a westerly QBO (WQBO) composite in Fig. 3 but an EQBO composite from a neutQBO run in Fig. 4. We conclude that the downward propagation of QBO anomalies is relatively unimportant for the polar response.

Figures $4 \mathrm{c}$ and $4 \mathrm{~d}$ show the quasi-steady response to EQBO tropical wind anomalies identical to that in Figs. $4 \mathrm{a}$ and $4 \mathrm{~b}$ in the lower stratosphere but with neutral QBO winds in the tropical upper stratosphere (cf. solid line in Fig. 2). In both the perpetual January run and the perpetual February run, the polar response to a QBO profile with realistic shear resembles the polar response to a QBO profile with weak upper stratospheric shear. Differences in the subtropical upper stratosphere are related to differences in the meridional circulation of the QBO in the absence of planetary waves. Upper stratospheric QBO anomalies appear less important than lower stratospheric QBO anomalies for the HT effect.

Figures $4 \mathrm{e}$ and $4 \mathrm{f}$ show the quasi-steady response to EQBO tropical wind anomalies identical in pattern but with lower stratospheric easterly anomalies 3 times stronger than that in Figs. $4 \mathrm{c}$ and $4 \mathrm{~d}$. The responses are qualitatively similar. Finally, we explore the response in the experiment on which we focus in section 4 and 5: the ensemble of runs in which $3 \times \mathrm{EQBO}$ winds are switched on (Figs. 5 and 6). In the first 15 days after branching, the tripole of tropical and subtropical lower stratospheric temperature anomalies associated with the meridional circulation of the QBO begins to develop. During days 16-30, the subtropical lower stratospheric warm anomaly spreads poleward of $30^{\circ} \mathrm{N}$ because of 


\section{EQBO-WQBO}
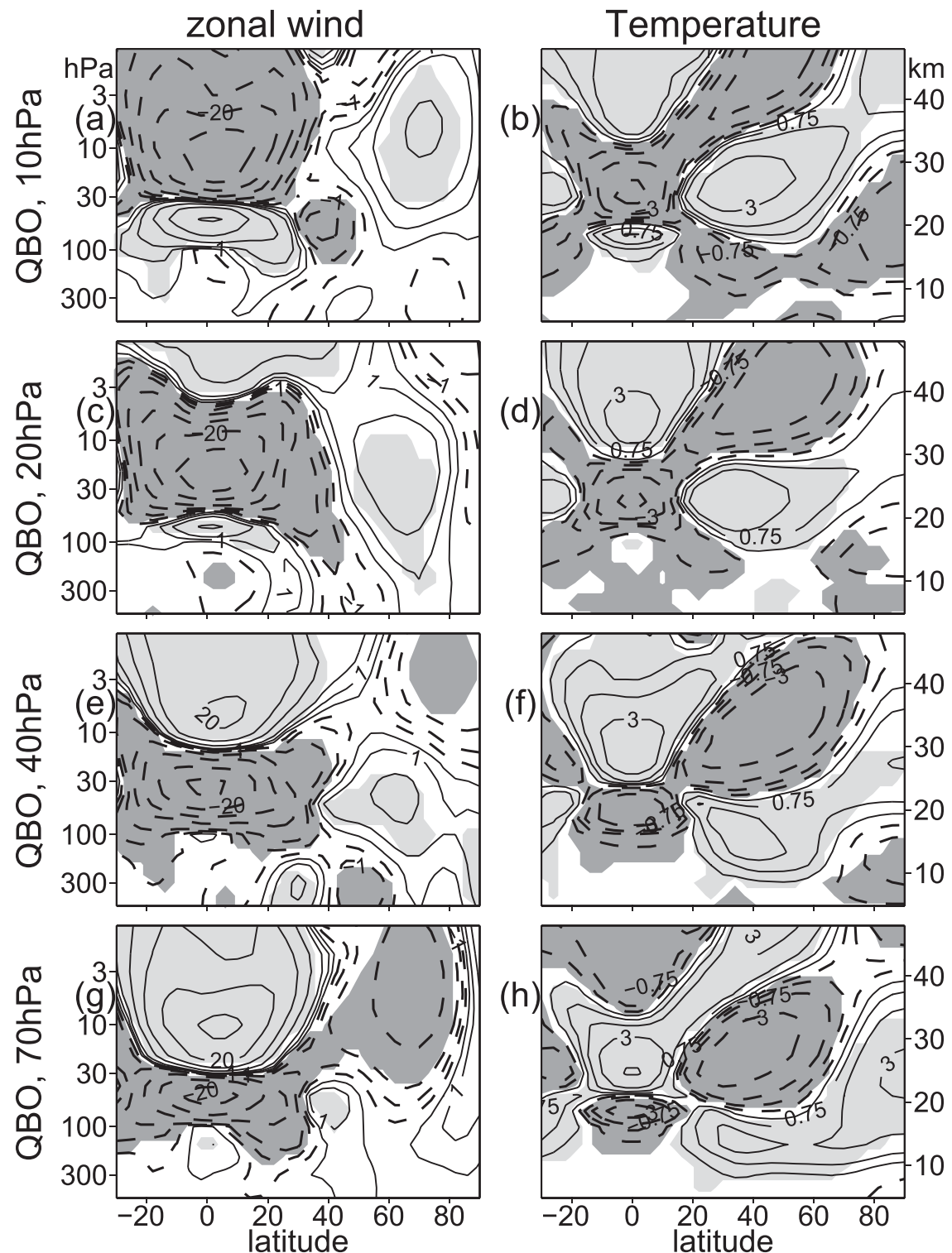

FIG. 3. Difference in (left) zonal wind and (right) temperature between EQBO and WQBO months for four different phases of a downward propagating QBO (EQBO - WQBO). Composites include the 85 months with the strongest westerly anomalies and 85 months with the strongest easterly anomalies, at $10,20,40$, and $70 \mathrm{hPa}$. Contours shown at (right) \pm 0.25 , $\pm 0.75, \pm 1.5, \pm 3$, and $\pm 6 \mathrm{~K}$ and (left) $\pm 0.33, \pm 1, \pm 2, \pm 5, \pm 10, \pm 20$, and $\pm 35 \mathrm{~m} \mathrm{~s}^{-1}$. Differences significant at the $95 \%$ level are shaded, and negative contours are dashed. A log-pressure height coordinate is shown on the right of this and similar ensuing plots.

adiabatic subsidence. Some of the upwelling that balances the midlatitude subsidence occurs over the pole (Fig. 6b), leading to a cold anomaly in the polar lowermost stratosphere and upper troposphere in Figs. 5b-d. Adiabatic subsidence near $30^{\circ} \mathrm{N}$ intensifies and extends further poleward after the first month, leading to the midlatitude lower stratospheric warm anomaly. Temperatures increase in this region in response to $\mathrm{EQBO}$ winds in the reanalysis as well (e.g., Fig. 1 of GH11a).

The upper stratospheric vortex begins to warm during days 16-30. Between days 15 and 60, the warm polar anomaly propagates downward with time, so that by day 60 the polar upper stratosphere is colder than in the neutQBO run. The downward propagation and cold upper 


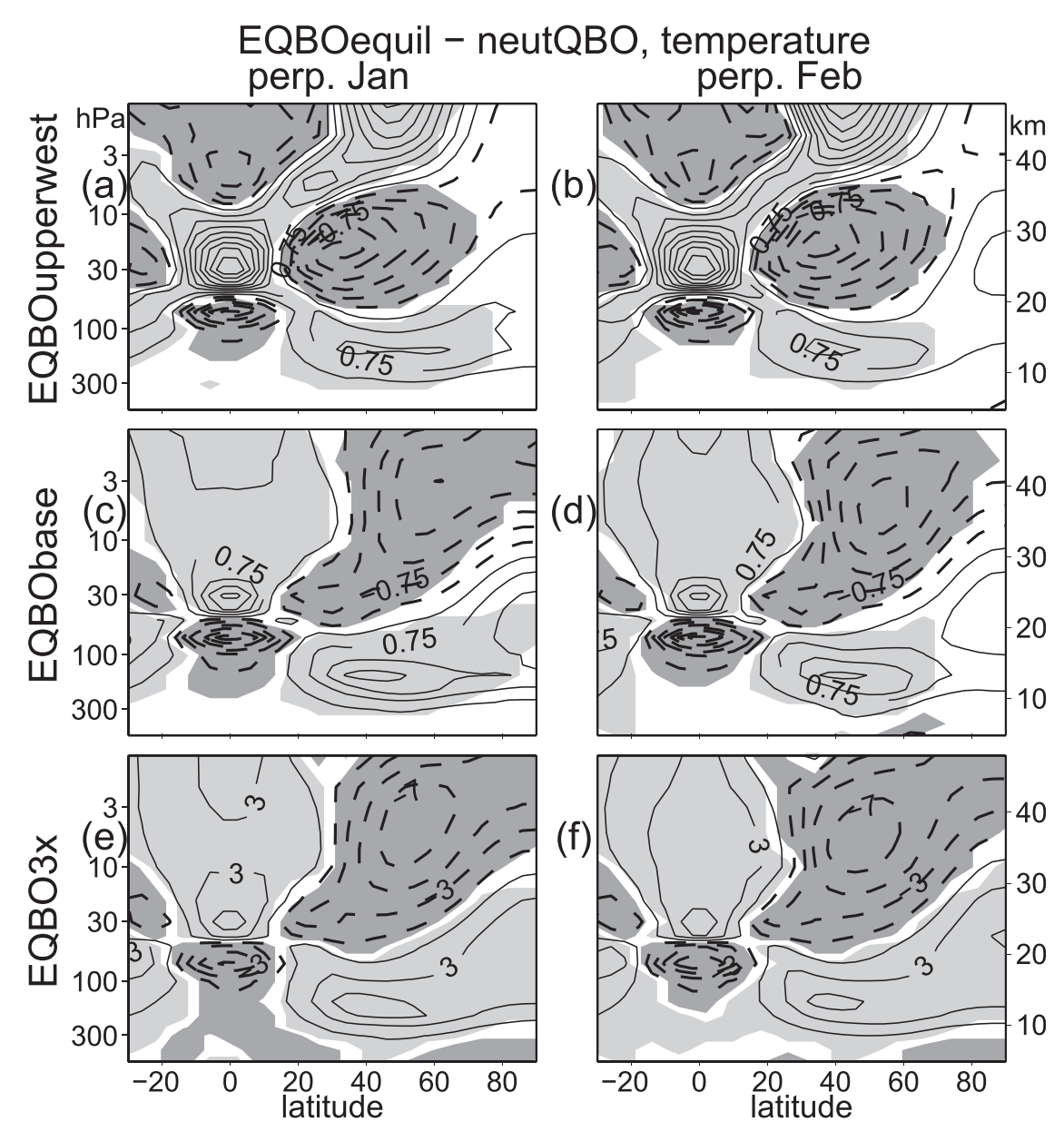

FIG. 4. Difference in temperature between the equilibrium EQBO cases and the control cases for the different EQBO wind profiles and calendar months (EQBO - neutQBO). Contours are shown at (a)-(d) $\pm 0.25, \pm 0.75, \pm 1.25, \ldots, \pm 4.25 \mathrm{~K}$ and (e),(f) $\pm 1, \pm 3, \pm 5, \pm 7$, and $\pm 9 \mathrm{~K}$. Differences significant at the $95 \%$ level are shaded, and negative contours are dashed.

stratosphere is reminiscent of Baldwin and Dunkerton (2001) and Dunkerton and Delisi (1985). In the last 60 days of the ensemble (Fig. 5d), the response is qualitatively similar to the quasi-steady response in Figs. 4e and $4 \mathrm{f}$. As in Kinnersley and Tung (1999), Kinnersley (1999), and Naito et al. (2003), the circulation is stronger in the winter hemisphere (i.e., the $\mathrm{NH}$ ).

In summary, the HT effect is qualitatively similar in all types of QBO runs. Sections 4 and 5 will now investigate the mechanism whereby the QBO influences the extratropics. Because the diagnostics are cleanest and easiest to understand in the ensemble of branch runs, we focus on how the switched-on $3 \times \mathrm{EQBO}$ winds weaken the vortex.

\section{Effect on tropospheric planetary wave driving}

The QBO can influence the troposphere through the circulation in thermal wind balance with anomalous equatorial winds (GH11a; GH11b). This change in the troposphere could influence tropospheric stationary planetary waves, and a change in these waves can subsequently affect the stratospheric vortex. Before we explore stratospheric mechanisms for the influence of the QBO on the polar vortex, we investigate whether the $\mathrm{QBO}$ can influence the polar vortex by first affecting the troposphere (e.g., Chen and Li 2007).

Garfinkel et al. (2010) and Fletcher and Kushner (2011) found that anomalies that constructively interfere with the climatological stationary waves (i.e., namely anomalies over the North Pacific and eastern Europe) weaken the vortex. EQBO leads to a low anomaly near Alaska (and Greenland) but a high height anomaly farther south (Figs. 7a,b). A similar anomaly is present in the reanalysis as well (e.g., Fig. 5 of Garfinkel and Hartmann 2010). To explore whether these anomalies would lead to a deeper planetary wave pattern, these height anomalies are added 


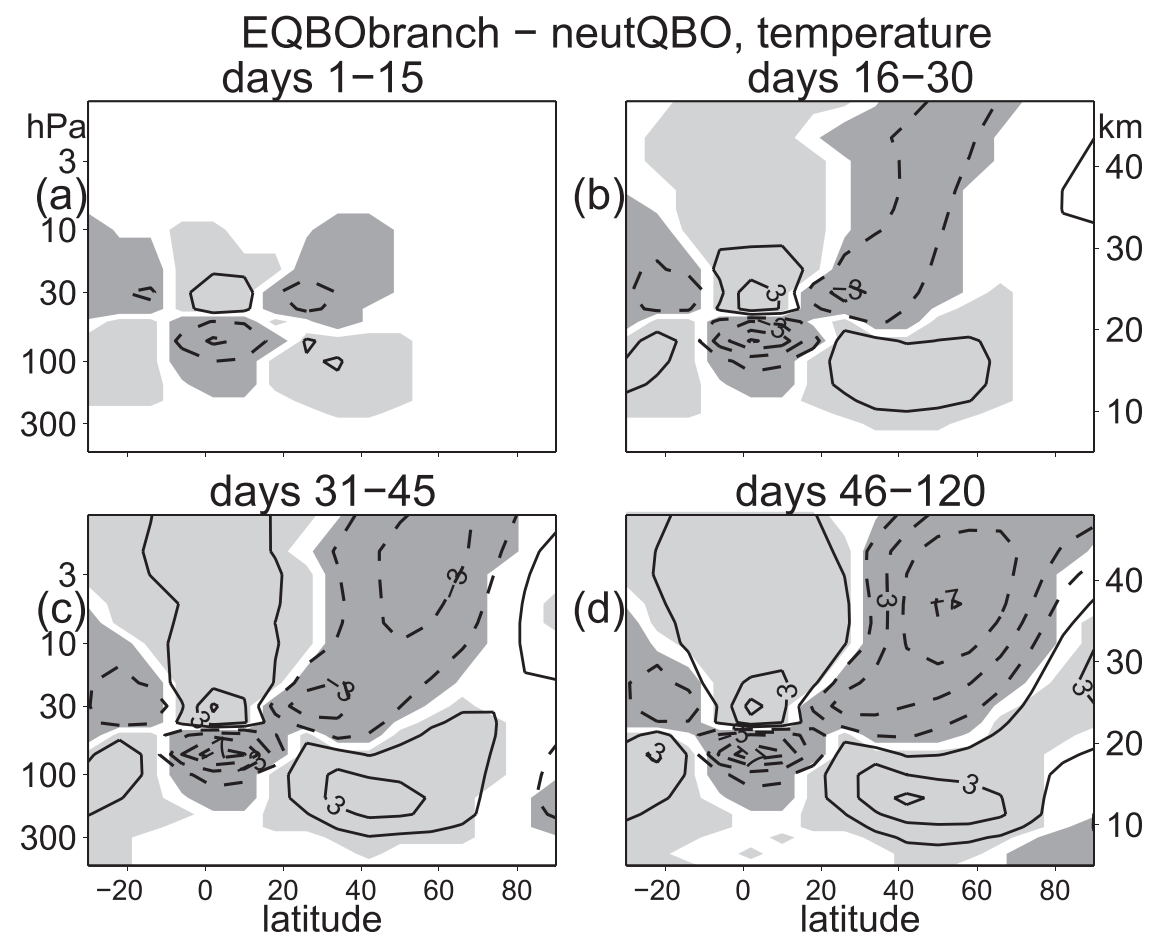

FIG. 5. Cross section of the difference in temperature between the February branch case and the common part of the February control case (EQBO - neutQBO) between days 1 and 15 , days 16 and 30, days 31 and 45, and days 46 and 120, after branching with $3 \times$ EQBO winds. Contours are shown at $\pm 1, \pm 3, \pm 5, \pm 7$, and $\pm 9 \mathrm{~K}$. All anomalies are a direct response to the QBO as we are comparing matched pairs of simulations. Regions where anomalies are unusual as compared to the control simulation at the $95 \%$ level are shaded. Negative contours are dashed.

onto the climatological stationary wave pattern in the neutQBO run. The tropospheric planetary waves with and without the geopotential height anomalies associated with EQBO are then compared. The magnitude of the climatological stationary waves (Fig. 7c) is essentially unchanged when the height perturbation associated with EQBO is added (Fig. 7d). Changes in upper tropospheric wave- 1 and wave- 2 vertical EPF are small. ${ }^{4}$ Changes in the planetary wave structure in the troposphere due to EQBO do not lead to enhanced upward EPF at the tropopause and to the observed weakening of the vortex. Rather, the mechanism through which the QBO affects the polar vortex in these simulations is internal to the stratosphere.

\footnotetext{
${ }^{4}$ If anything, stationary wave magnitude is actually decreased, which would imply a slightly stronger vortex for EQBO. Garfinkel and Hartmann (2008) found that differences in wave-1 and wave-2 $\mathrm{EPF}$ are not significant below the tropopause in reanalysis data. Future investigation is needed to understand whether WQBO winds might affect tropospheric planetary waves.
}

\section{Effect on the stratospheric wave propagation}

We now explain the mechanism whereby an easterly wind anomaly in the lower equatorial stratosphere affects the polar vortex. We first present EPF vectors and convergence (section 5a) to diagnose anomalies of Rossby wave propagation in response to the QBO. We then interpret the EPF anomalies using linear theory (section $5 b$ ). We focus our attention on two regions: the midlatitude lower stratosphere and the subpolar upper stratosphere. Overall, we will show that while linear QG theory applied to Rossby wave propagation can explain how the polar vortex is modulated by the QBO, the HT mechanism cannot.

\section{a. Influence of finite-amplitude waves}

Figure 8 shows that the location of EPF convergence and divergence changes as we branch with EQBO winds. During days 1-15 after branching two dipoles of EPF convergence develop.

(i) In the lowermost stratosphere, EPF convergence at the flanks of the $\mathrm{QBO}$ relaxation region (i.e., 


\section{EQBObranch-neutQBO, $\psi^{* \star}$}
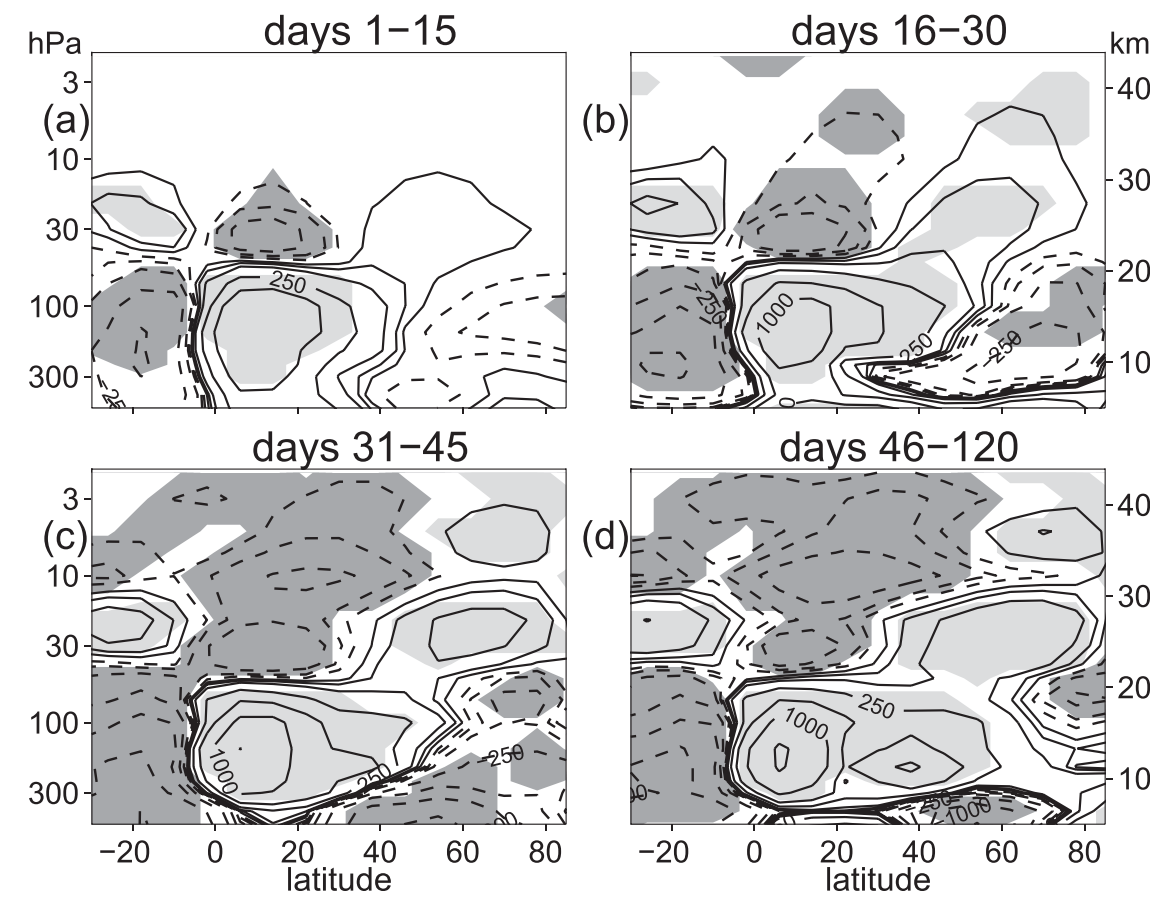

FIG. 6. As in Fig. 5, but for transformed Eulerian mean streamfunction anomalies. Contours are shown at $\pm 75, \pm 250, \pm 500, \pm 1000$, and $\pm 2000 \mathrm{~kg} \mathrm{~m}^{-1} \mathrm{~s}^{-1}$.

$20^{\circ}-30^{\circ} \mathrm{N}, 50-100 \mathrm{hPa}$ ) is increased while wave convergence closer to the equator is reduced. The reduction in wave convergence near the equator is nearly twice as large, but qualitatively similar, if we consider the full (as opposed to the QG) EPF (not shown). Waves do not propagate as far toward the equator in the lower stratosphere in the ensemble of EQBO runs as in the neutQBO run.

(ii) Even though the subtropical upper and middle stratosphere (i.e., $20^{\circ}-30^{\circ} \mathrm{N}, 1-10 \mathrm{hPa}$ ) is not directly affected by the EQBO anomaly, a dipole of EPF convergence anomalies develops in the upper stratosphere whereby EPF convergence is decreased near $20^{\circ}-30^{\circ} \mathrm{N}$ and increased near $40^{\circ}-50^{\circ} \mathrm{N}$.

Overall, Rossby waves cannot reach as far equatorward in the EQBO ensemble as they can in the neutQBO run. This change in Rossby wave propagation influences zonal wind and temperature throughout the extratropics:

(i) Westerlies intensify between $20^{\circ}$ and $30^{\circ} \mathrm{N}$ in the upper stratosphere, as one might expect from the EPF divergence in that region. (This occurs even in the presence of realistic upper stratospheric westerlies; see Fig. $3 \mathrm{~g}$ near $20^{\circ}-30^{\circ} \mathrm{N}, 3-10 \mathrm{hPa}$ ).

(ii) In the lower subtropical stratosphere, increased EPF convergence (a) causes an easterly acceleration that shifts poleward the boundary of the easterlies associated with the QBO by $\sim 5^{\circ}$ and (b) forces an anomalous residual circulation with subsidence near $30^{\circ}-50^{\circ} \mathrm{N}, 100 \mathrm{hPa}$ (Fig. 6). The downward motion near $30^{\circ}-50^{\circ} \mathrm{N}$ causes a poleward extension of the temperature anomaly associated with the QBO's meridional circulation in thermal wind balance (Fig. 5a).

(iii) However, little change is observed in the polar vortex region (cf. Fig. 5a).

Waves converge anomalously near the flanks of the QBO even at the earliest stages following branching and thereby influence the extratropics.

During days 16-30 after branching, the changes that occur between days 1 and 15 intensify and spread poleward (Figs. 8, 5b, and 6b). In particular,

(i) Rossby wave propagation (and the associated EPF convergence) in the lower stratosphere is modulated significantly. The increase of EPF convergence reaches near $50^{\circ} \mathrm{N}$, which leads to warmer midlatitude lower stratospheric temperatures and poleward extension of the tropical easterlies beyond the QBO relaxation region (see section $5 b$ ). In contrast, EPF convergence in the deep tropics equatorward of $10^{\circ} \mathrm{N}$ is reduced.

(ii) As during days 1-15 after branching, fewer Rossby waves propagate into the subtropical upper stratosphere even though the winds in the tropical upper 


\section{height at 500hpa}

EQBObranch-neutQBO, month 2

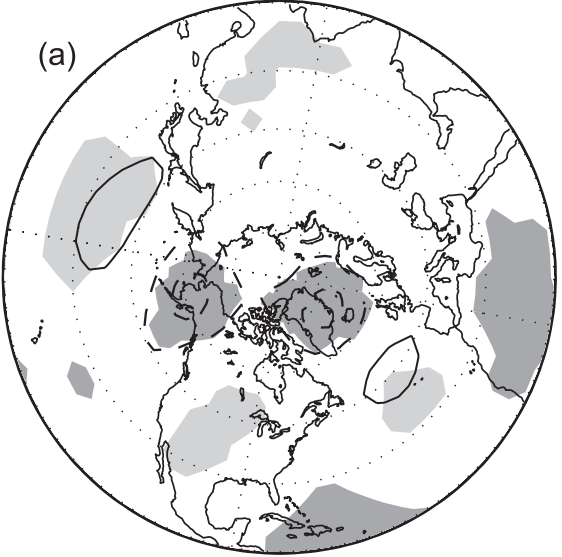

EQBObranch-neutQBO, month 4

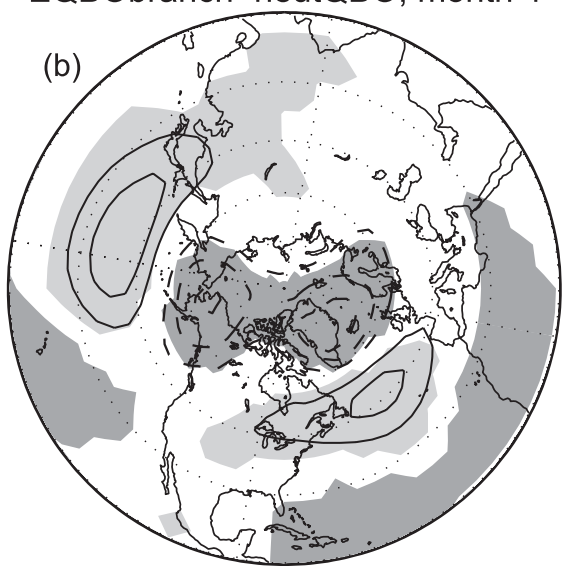

Low wavenumber, neutQBO

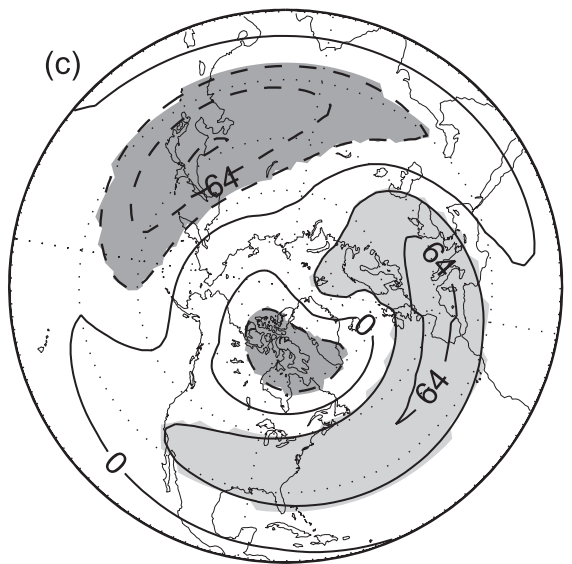

Low wavenumber, EQBObranch, month 4

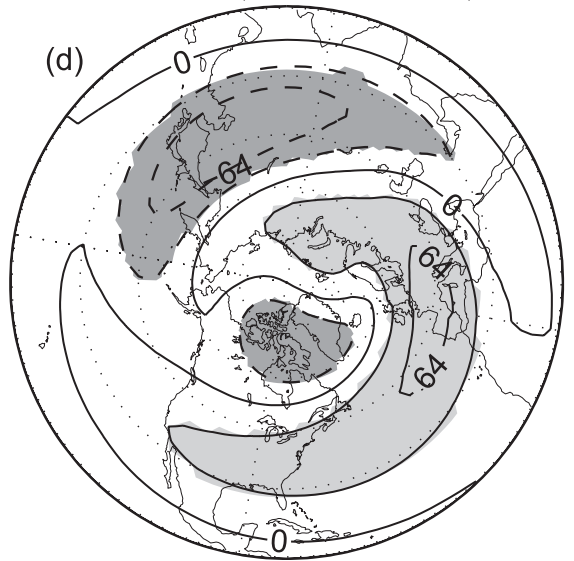

FIG. 7. Height anomalies after branching in perpetual February with $3 \times$ EQBO winds: month (a) 2 and (b) 4. Contour interval is $20 \mathrm{~m}$, the zero contour is omitted, and significant regions at the $95 \%$ level are shaded (EQBO - neutQBO). The low wavenumber eddy height field (i.e. wavenumber 1 plus wavenumber 2) at $500 \mathrm{hPa}$ for (c) the neutral QBO run and (d) the EQBO branch run in month 4. Contour interval is $32 \mathrm{~m}$, and regions with large zonal asymmetries are shaded. Negative contours are dashed.

stratosphere are not directly affected by the QBO relaxation. The EPF convergence anomaly near $50^{\circ} \mathrm{N}, 3 \mathrm{hPa}$ during days $1-15$ can therefore intensify and spread poleward. Even though total extratropical heat flux at the tropopause is not significantly changed, reduced wave propagation into the subtropical middle and upper stratosphere leads to enhanced EPF convergence near the vortex. A residual circulation therefore develops in the upper stratosphere whereby polar cap temperatures increase while midlatitude temperatures decrease.

By days 31-45, the anomalies in EPF convergence qualitatively resemble their equilibrium values. In the lower stratosphere, changes are qualitatively similar to days 16-30. As the upper stratospheric vortex weakens, subpolar vertical EPF cannot reach as high into the stratosphere because of the dynamical feedbacks commonly seen during a SSW (e.g., Limpasuvan et al. 2004; see also Dunkerton and Delisi 1985). As a result, the subtropical upper stratospheric divergence anomaly spreads poleward and waves break lower in the stratosphere than in the neutQBO run, which then leads to downward propagation of the polar temperature anomalies. ${ }^{5}$

Figure 9 decomposes the EPF into wavenumber components for days $16-30$ and $46-120$ after branching. The effect near the polar vortex is dominated by wave 1 (especially stationary wave 1 ). In contrast, wave 1 has little effect in the subtropics near $100 \mathrm{hPa}, 40^{\circ} \mathrm{N}$ where waves $2-13$, and

\footnotetext{
${ }^{5}$ In the troposphere, EPF (and in particular momentum flux) anomalies cause, and then positively feed back onto the poleward shift of the tropospheric jet, as discussed in GH11a and GH11b.
} 
EQBObranch-neutQBO, EPF and divergence

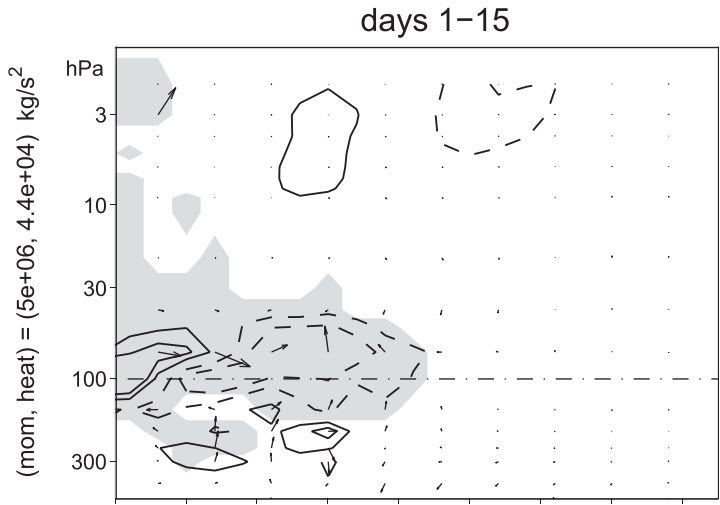

days $16-30$

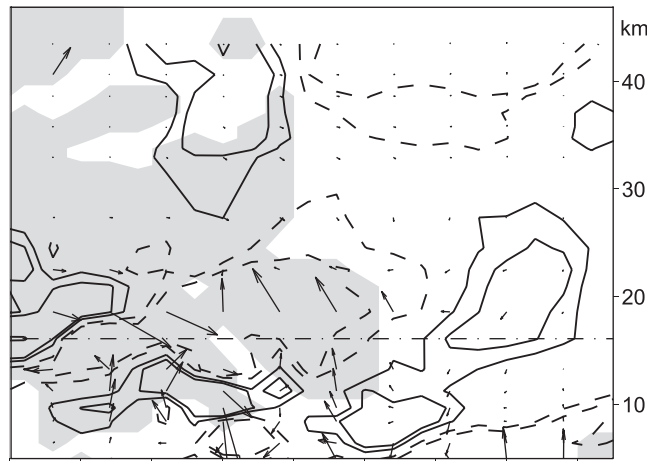

days $31-45$
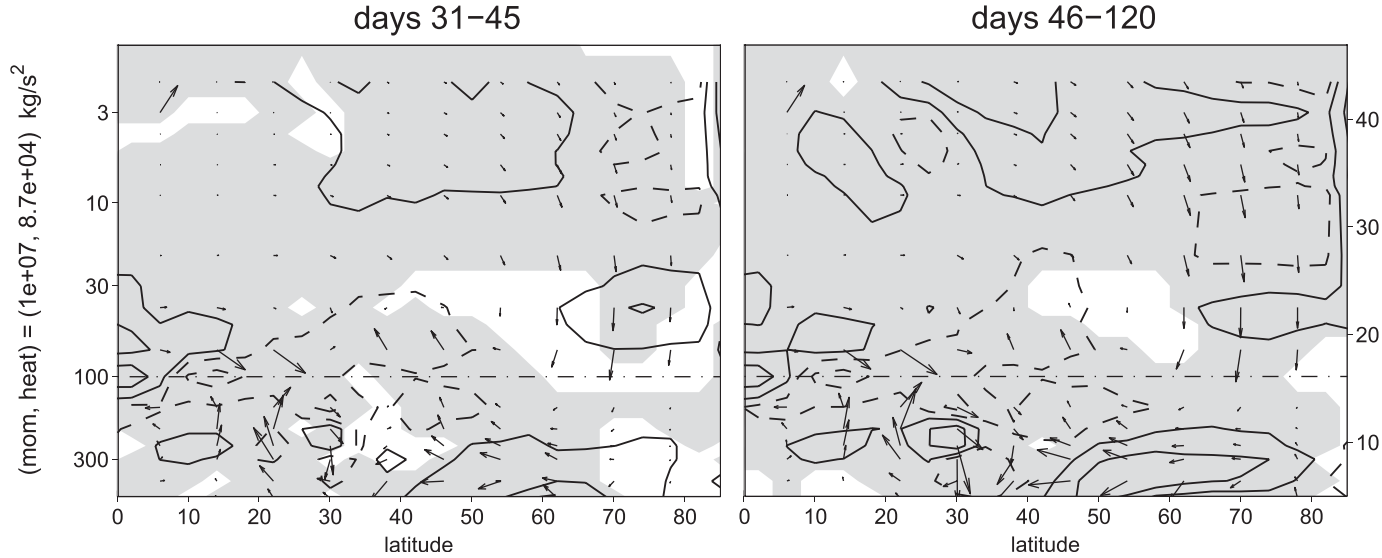

FIG. 8. EPF (all wavenumbers and stationary plus transient) cross section averaged between days 1 and 15 , days 16 and 30, days 31 and 45, and days 45 and 120, after branching with $3 \times$ EQBO winds (EQBO - neutQBO). EPF arrow lengths are multiplied by a factor of 5 in the stratosphere. For clarity, the arrow scaling in (top) the first month after branching is half of that in (bottom) the subsequent three months. Contours of the divergence of the EPF are shown at $\pm 0.12, \pm 0.25, \pm 1$, and $\pm 2 \mathrm{~m} \mathrm{~s}^{-1}$ day $^{-1}$ in the top row; in the bottom row, the $\pm 0.12 \mathrm{~m} \mathrm{~s}^{-1} \mathrm{day}^{-1}$ contour is omitted. A reference arrow for the stratosphere is located in the top-left corner of the plots, and its magnitude is on the far left. All anomalies are forced by the QBO as we are comparing matched pairs of simulations. Regions where either component of the EPF is unusual as compared to the control simulation at the $95 \%$ level are shaded. Negative contours are dashed.

in particular transients of wavenumbers $4-13$, are dominant. Synoptic wave breaking is influenced by the QBO even in the first month after branching (e.g., GH11b).

In summary, EPF convergence in the subtropical lower stratosphere in the flanks of the QBO relaxation region is enhanced, and the effect spreads poleward throughout the run. Wave-1 EPF convergence in the subpolar stratosphere is enhanced while wave propagation into the subtropical upper stratosphere is reduced. Once the vortex begins to weaken (i.e., after day 30), waves break lower in the stratosphere than in the neutQBO run, leading to a downward propagating warm polar anomaly. Section $5 \mathrm{~b}$ will interpret these changes in the context of linear theory.

\section{b. Comparison with linear diagnostics}

We now show that linear theory can explain how the easterly wind anomaly in the lower equatorial stratosphere affects Rossby wave propagation. We first focus on changes of the index of refraction (Fig. 10) in the subtropical lower stratosphere, in the midlatitude middle and upper stratosphere, and in the polar stratosphere. Figure 10a shows the index of refraction for the mean state in the neutQBO run, while Fig. 10b shows the index of refraction if the axisymmetric meridional circulation of the QBO in thermal wind balance (as given in GH11a) is added onto the mean state of the neutQBO run. Figures 10c-f show anomalies of the index of refraction after branching with EQBO winds. EQBO winds affect the index of refraction in the following regions.

(i) In the subtropical lowermost stratosphere, the zero wind line moves poleward after branching with EQBO winds, consistent with the HT mechanism (horizontal dashes vs filled stars in Fig. 10). 


\section{EQBObranch-neutQBO, EPF and divergence}
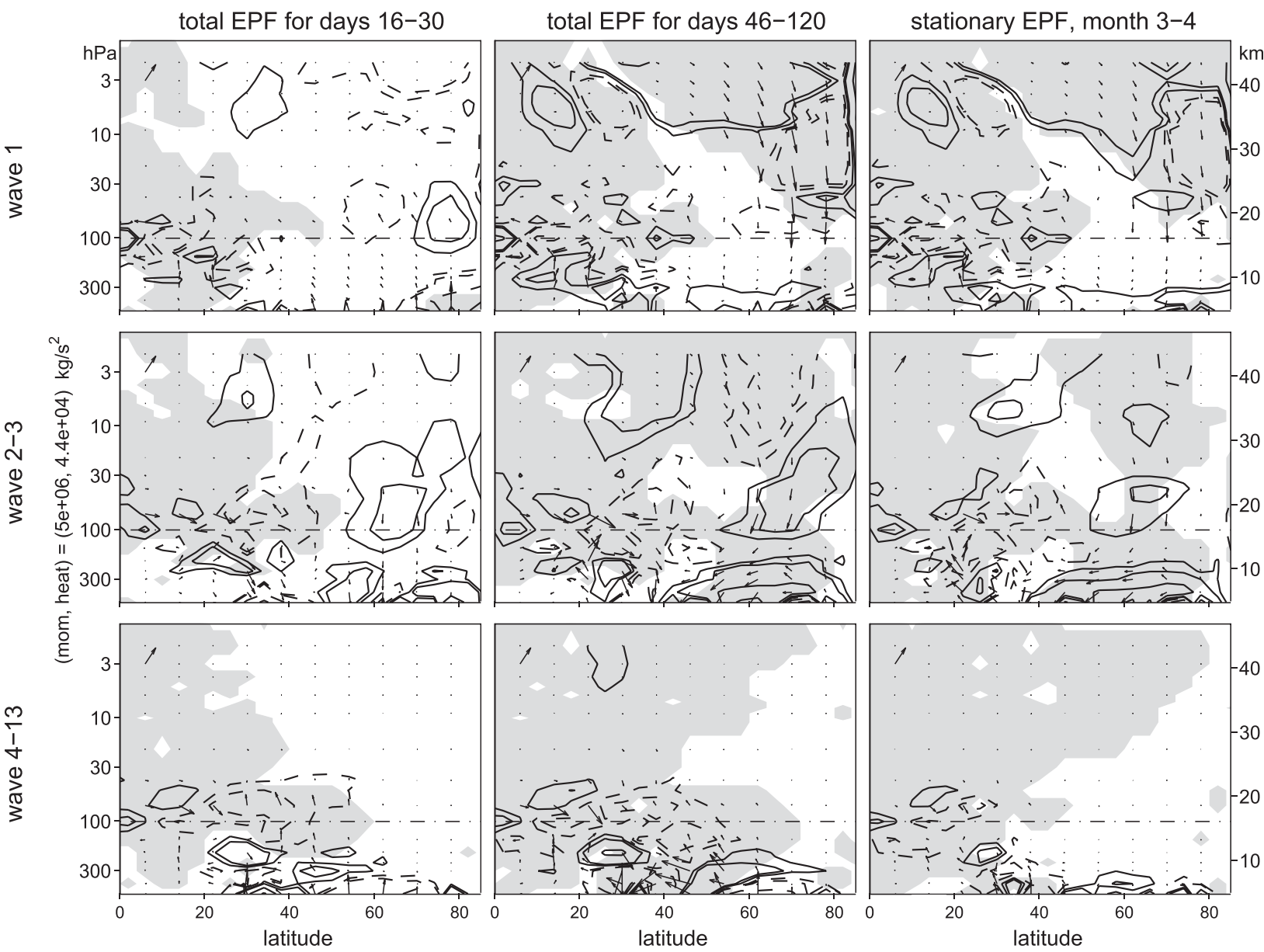

FIG. 9. EPF cross section (left) for days 16-30 and (middle),(right) well after branching with $3 \times$ EQBO winds [(middle) transient and stationary; (right) stationary only], for (top) wave 1, (middle) waves 2 and 3, and (bottom) waves 4-13. Plotting conventions are as in the top row of Fig. 8.

Simultaneously, the region near $100 \mathrm{hPa}, 40^{\circ} \mathrm{N}$ from which waves are climatologically refracted away shrinks in latitude and height. ${ }^{6}$ These changes lead to enhanced Rossby wave convergence near $30^{\circ}-40^{\circ} \mathrm{N}$ in the lowermost stratosphere. Wave feedbacks can then amplify these initial changes in the critical lines, so that the effect is strongest and reaches farthest poleward later in the run (cf. Figs. 10c and 10f).

(ii) The index of refraction near $50^{\circ} \mathrm{N}, 5-50 \mathrm{hPa}$ decreases upon branching with EQBO winds (e.g., Fig. 10d). A decrease in index of refraction in this region explains the dipole of EPF anomalies in the

\footnotetext{
${ }^{6}$ Changes in this region were not hypothesized by HT. Nevertheless, changes in EP flux in this region follow linear theory.
}

upper stratosphere in days 1-30 after branching. That is, wave propagation from subpolar latitudes to subtropical latitudes is suppressed, leading to more wave convergence near the vortex. As this change in index of refraction is vital for the weakening of the vortex in the first month after branching, the contribution of each term constituting the index of refraction is isolated. A reduction of $\overline{q_{y}}$, and in particular of $\left[(\bar{u} \cos \phi)_{\phi} / a \cos \phi\right]_{\phi}$, is responsible for the reduced index of refraction values (not shown). Changes in zonal wind in this region are due to the axisymmetric circulation of the QBO (i.e., index of refraction is reduced in Fig. 10b as well), as easterly zonal wind anomalies at the equator require westerly anomalies near $30^{\circ} \mathrm{N}$ to maintain geostrophic balance (see GH11a). Gradients of this westerly zonal wind anomaly lead to reduced equatorward 


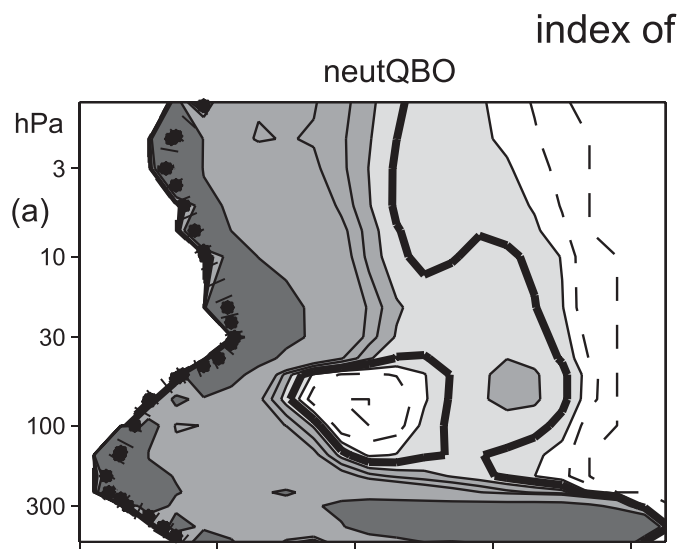

(b)

\section{neutQBO+ $+\triangle \mathrm{EQBO}$}
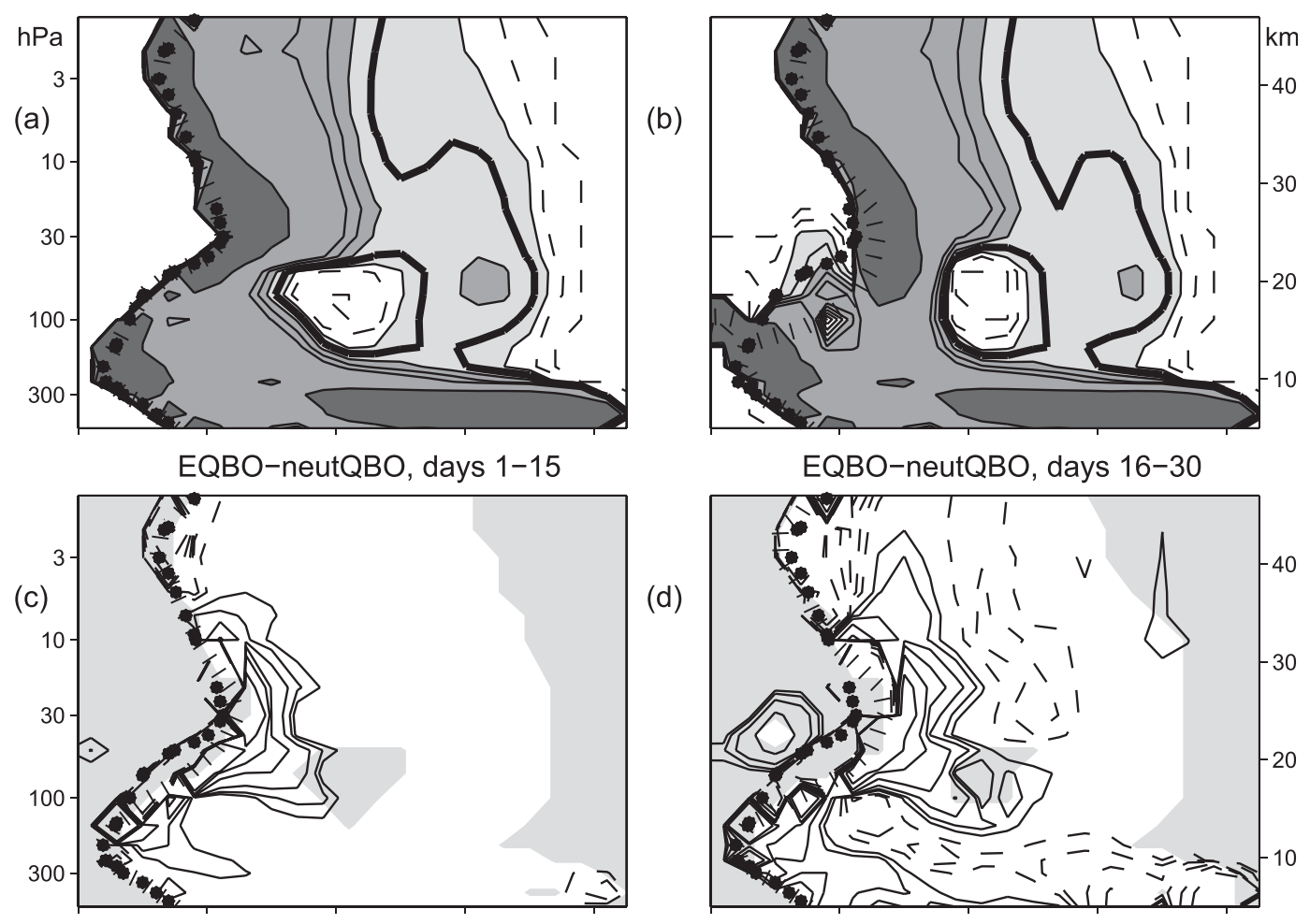

EQBO-neutQBO, days 16-30

(d)

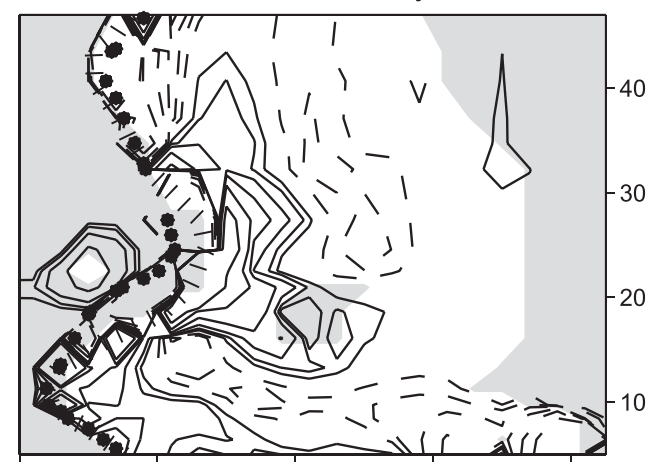

EQBO-neutQBO, days 31-45

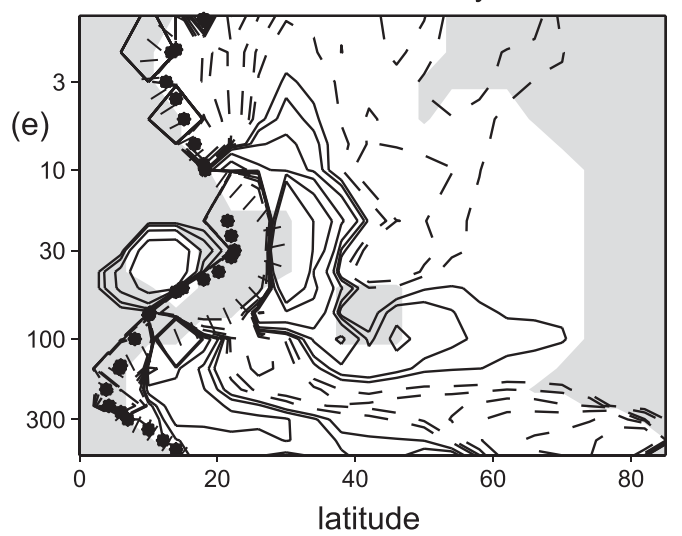

(f)

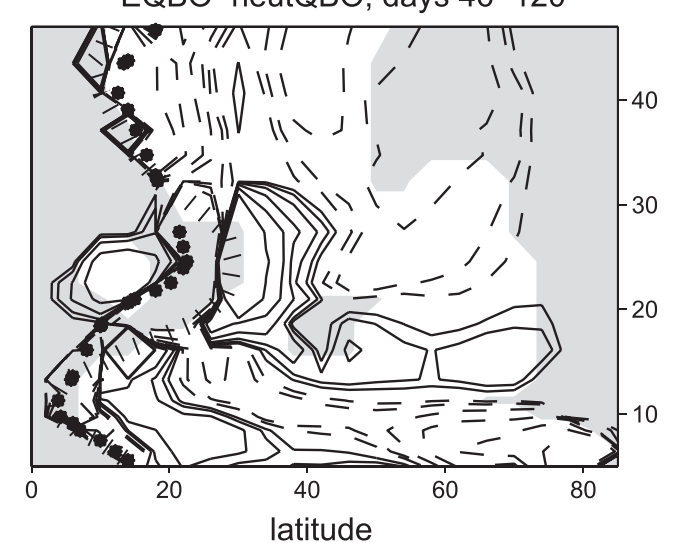

FIG. 10. Index of refraction multiplied by the earth's radius squared $\left(n^{2} a^{2}\right)$ for stationary wavenumber 2 (a) in the neutQBO run and (b) in the neutQBO run perturbed by the meridional circulation of the QBO as defined in GH11a. Contours are shown at $-20,-10,0,10,20,30,40,100,200$, and 300 , and higher values are in darker gray. The 10 contour is bolded to show clearly the decrease in index of refraction in the midstratosphere near $50^{\circ} \mathrm{N}$. (c)-(f) As in (b), but anomalies in the ensemble of runs after branching with $3 \times$ EQBO winds (EQBO - neutQBO). Contours are shown at $\pm 2, \pm 4, \pm 8, \pm 16, \pm 32$, and \pm 64 . Regions of wave evanescence are shaded. The zero wind line of the neutQBO run is marked by filled circles in all subplots. The zero wind line of each subplot is shown with perpendicular dashes. Negative contours are shown with parallel dashes.

propagation and a dipole of EPF convergence in the upper stratosphere. Thus, linear theory suggests that the mean meridional circulation of the QBO, not the subtropical critical line, is responsible for the weakening of the vortex. (iii) After the first month and the initial weakening of the vortex, the polar reflecting surface moves downward and equatorward. The expansion of the polar region of negative index of refraction hinders subpolar vertical EPF from reaching as high in the 
stratosphere. Changes in the subtropical critical line do not explain this effect.

In summary, changes in the index of refraction, but not in the subtropical critical line (i.e., the HT mechanism), are needed to explain the QBO's effect near the vortex. Nevertheless, index of refraction arguments do not separate the impacts of meridional versus vertical wave propagation changes, accurately predict which zonal wavenumbers produce the changes, or account for wave tunneling (Harnik and Lindzen 2001). It is conceivable that these effects may be important for the stratospheric response. We therefore examine wave propagation in the idealized QG model described in section 2. Specifically, we will show that the linear QG model can simulate the wave propagation changes in WACCM, implying that linear theory is important for the response in WACCM as well.

Figure 11 shows the evanescent regions (shading) and changes in the stationary (left) meridional and (right) vertical wavenumbers after branching with EQBO winds for wave 1 . Figure 12 is like Fig. 11 but for wave 2 . Figure 13 shows EPF anomalies from the QG model during days 16-30 and 46-120 for waves 1 and 2. We discuss the response in the QG model in the subtropical lower stratosphere and upper stratosphere-polar vortex.

(i) Subtropical lower stratospheric meridional wavenumbers $l^{2}$ increase, the MWG moves farther poleward, and the barrier to wave propagation near $40^{\circ} \mathrm{N}$, $70 \mathrm{hPa}$ shrinks, after imposing EQBO winds. These changes are stronger for wave 2 than wave 1 (cf. Figs. 11 and 12). Associated with the change in the MWG and meridional wavenumber for wave 2 is a poleward shift in EPF divergence because waves cannot propagate as far equatorward as they can in the neutQBO run (Fig. 13). Transients of wavenumber 4-6 are also blocked from propagating as far equatorward under $\mathrm{EQBO}$ as they can in the neutQBO run (not shown). All of these changes are consistent with the changes in the index of refraction and with the EPF in WACCM shown in Figs. 8 and 9.

(ii) In the upper stratosphere near $50^{\circ} \mathrm{N}, 5-50 \mathrm{hPa}$, a negative meridional wavenumber anomaly develops in the first 30 days, consistent with the decreased index of refraction locally (Fig. 10d). This negative anomaly expands to encompass the polar stratosphere and becomes associated more with the vertical wavenumber, consistent with enhanced EPF convergence over the vortex and reduced EPF convergence in the subtropics during days 16-30. The negative anomaly then intensifies and propagates downward as the vertical reflecting surface descends. All of these changes are stronger for wave 1 than for wave 2 , and stationary wave 2 leads, if anything, to an acceleration of the vortex (Figs. 13c,d, middle row of Fig. 9). As the vertical reflecting surface descends after the first month, a region of EPF divergence develops in the upper stratosphere because waves cannot access the upper stratosphere (Fig. 13b). The anomalous background state experienced by the upward propagating waves leads to weakening of the upper stratospheric vortex in the first month after branching and to downward propagation of the anomaly in months two through four after branching.

Qualitative agreement between the linear QG model and the nonlinear WACCM (cf. Figs. 9 and 13) is strongly suggestive that linear theory can explain the response to the QBO in WACCM, although the evolution of the entire wind distribution is only quasi-linear because induced zonal wind changes cause the wave convergence to move.

\section{Effect of shear and upper stratospheric winds}

Sections 4 and 5 have shown that linear theory can explain the effect of lower stratospheric easterlies on the polar vortex. Specifically, the axisymmetric circulation associated with easterly winds at the equator near $50 \mathrm{hPa}$ creates a barrier to wave propagation from subpolar latitudes to midlatitudes in the middle and upper stratosphere (e.g., less equatorward EPF near $50^{\circ} \mathrm{N}$ ), which leads to enhanced wave convergence in the polar vortex region. But the experiment examined was highly idealized: it was an ensemble of branch runs with $3 \times \mathrm{EQBO}$ winds in the lower stratosphere only. Even though section 3 showed that the HT effect was qualitatively similar for more realistic experimental configurations, we wish to discuss the effect of QBO profiles of realistic strength and with realistic shear in the mid- and upper stratosphere on stratospheric wave propagation.

Figure 14 shows EPF cross sections for the perpetual January quasi-steady equilibrium runs discussed in section 3 . The response to default and $3 \times \mathrm{EQBO}$ winds qualitatively resembles that shown in Fig. 8. The response to an EQBO profile with realistic upper stratospheric shear is similar through much of the stratosphere (including near the polar vortex) but differs in the subtropical upper stratosphere. Even though there are westerlies in this region and the critical line moves equatorward in the presence of shear, equatorward EPF propagation is reduced. The difference is due largely to wave 1 (not shown); wave 2 and higher Rossby waves only weakly propagate into this region, as might be expected from Charney and Drazin (1961). This wave-1 effect is inconsistent with the HT mechanism as the changes in the critical line would imply that more Rossby waves should propagate into this region. 

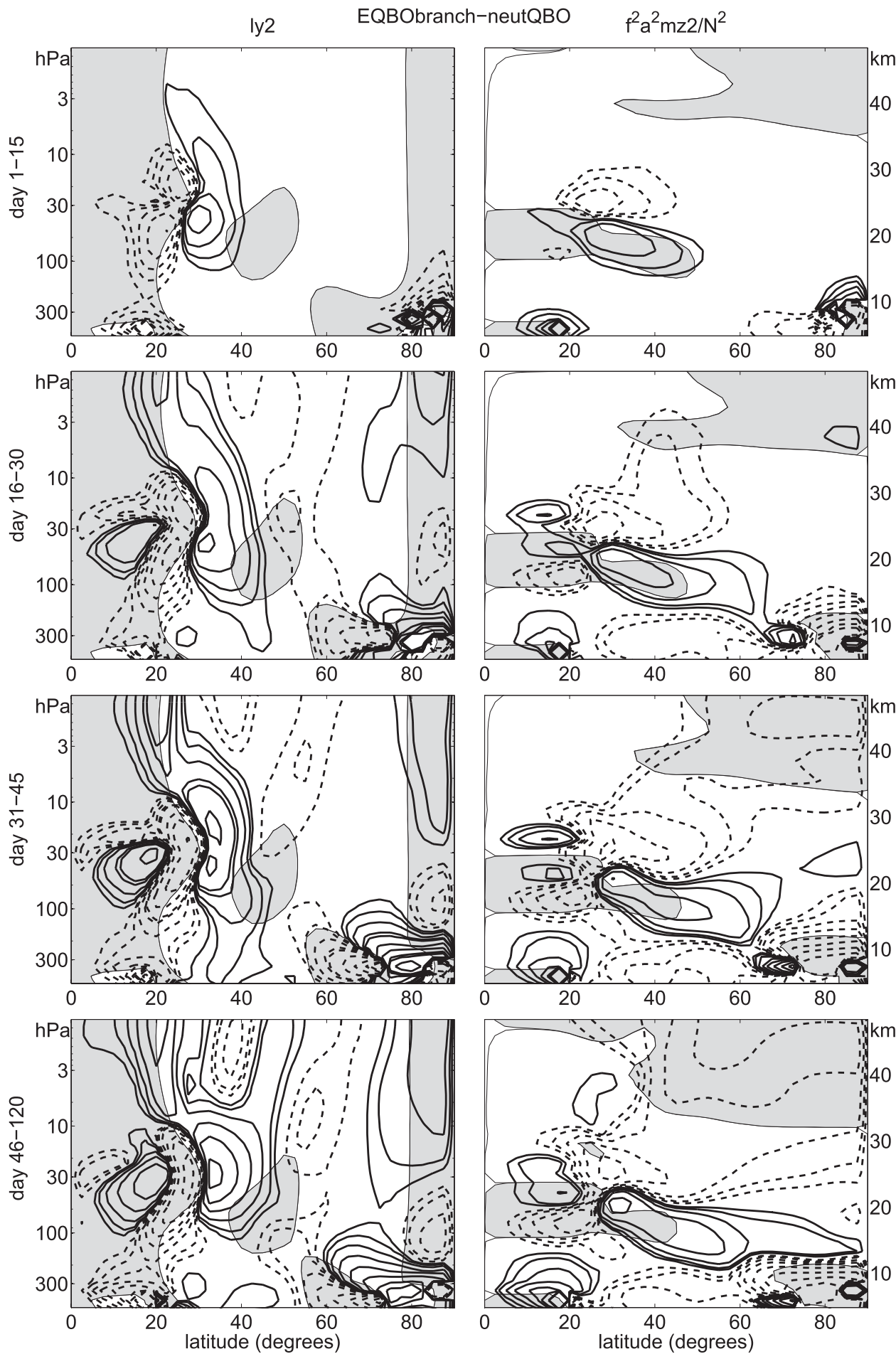

FIG. 11. Stationary wave-1 (left) meridional and (right) vertical wavenumber anomalies in the $3 \times$ EQBO branch run from the QG model (EQBO - neutQBO). Regions of evanescent waves are shaded. Contours are shown at $\pm 1, \pm 2, \pm 4, \pm 8, \pm 16, \pm 32$, and \pm 128 (dimensionless). Negative contours are dashed. 

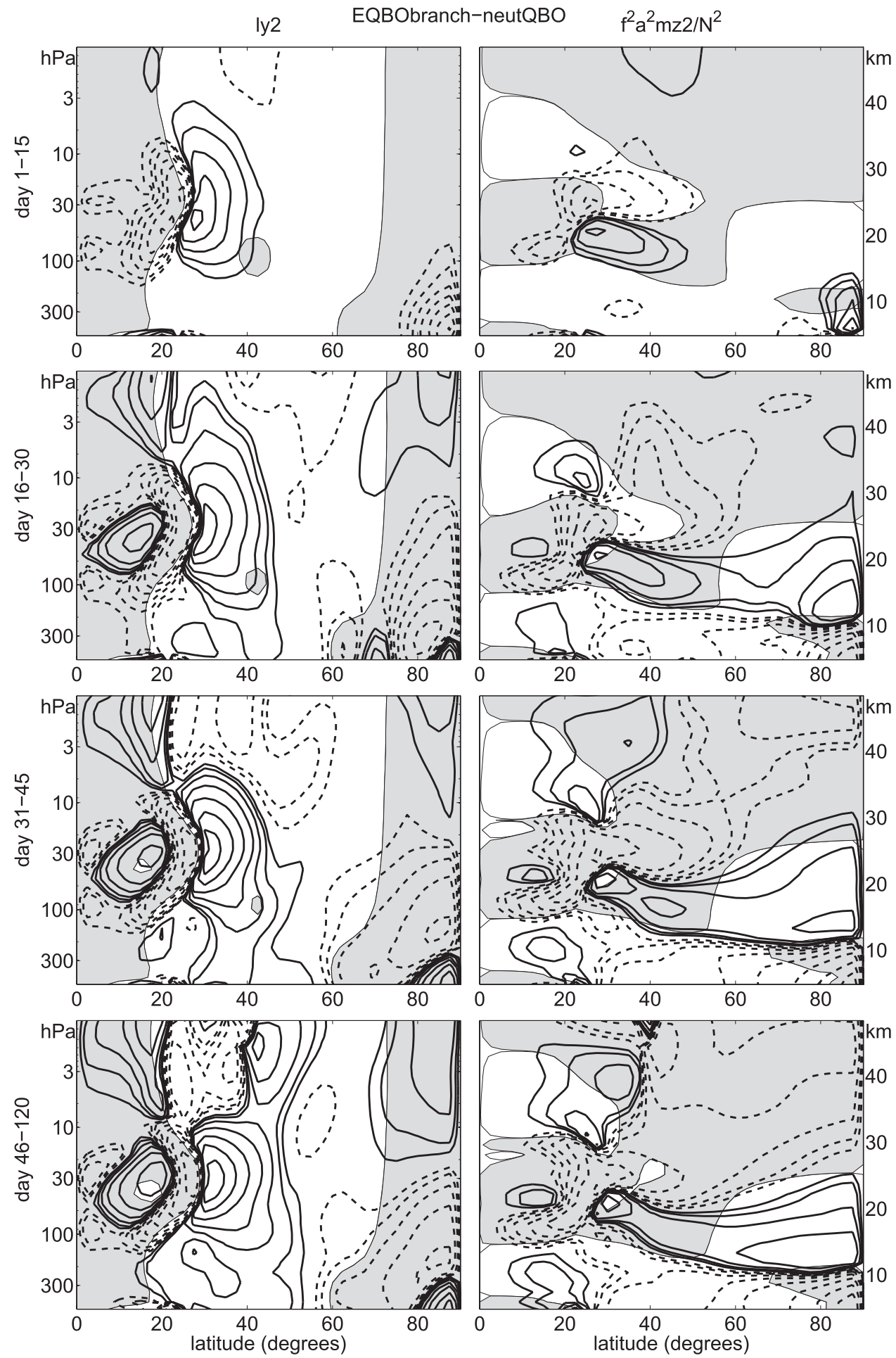

FIG. 12. As in Fig. 11, but for wave 2.

To explain why wave-1 propagation into the subtropical upper stratosphere is reduced even as the critical line moves equatorward, we turn to the index of refraction. Although index of refraction values increase equatorward of $20^{\circ} \mathrm{N}$, they decrease near $40^{\circ} \mathrm{N}$ in the upper stratosphere (above $50 \mathrm{hPa}$ ) in response to upper stratospheric westerlies (not shown). Because equatorward wave propagation near $40^{\circ} \mathrm{N}$ is suppressed, EPF convergence between 

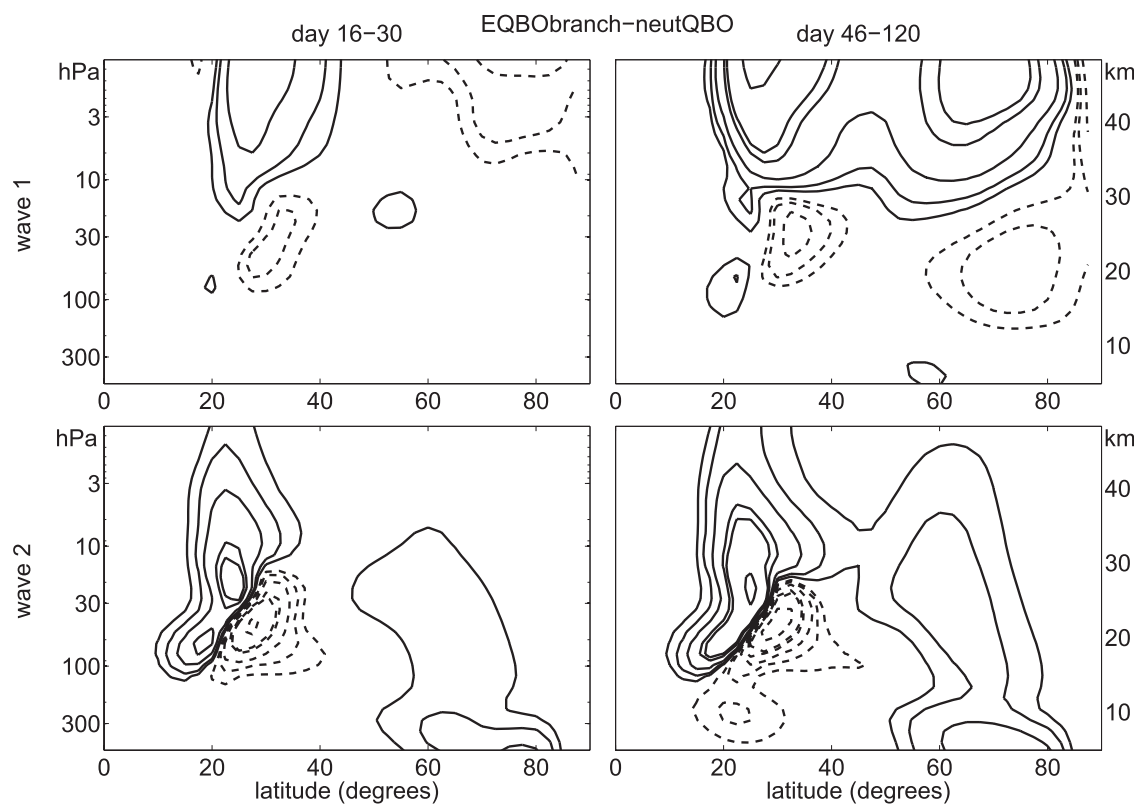

FIG. 13. (top) Wave-1 and (bottom) wave-2 EPFD in the stationary QG model during days (left) 16-30 and (right) 46-120 after branching (EQBO - neutQBO). Units of EPFD are $\mathrm{m} \mathrm{s}^{-1}$ day $^{-1}$. The contour interval is linear $(1,2,3, \ldots)$, but the precise values are arbitrary because we are using a linearized model. Negative contours are dashed.

$20^{\circ}$ and $40^{\circ} \mathrm{N}$ significantly decreases. The reduced index of refraction values near $40^{\circ} \mathrm{N}$ are caused by reduced $q_{y}$ \{in particular from $\left.\left[(u \cos \varphi)_{\varphi} / a \cos \varphi\right]_{\varphi}\right\}$ near the transition from tropical and subtropical westerlies to subpolar easterlies (not shown, but see Fig. $3 \mathrm{~g}$ near $40^{\circ} \mathrm{N}$, $3 \mathrm{hPa}$ ). Index-of-refraction arguments can therefore explain the difference in response between the case with upper-stratospheric westerlies and the case with neutral upper-stratospheric winds. Finally, we have also examined this case in the linearized QG model (not shown). The subtropical vertical reflecting surface expands downward in response to a sheared QBO. The reflecting surface appears when the wind shear $\partial u / \partial z$ is negative (Perlwitz and Harnik 2003), and in the sheared case the vertical zonal wind shear is negative in the tropics between 2 and $10 \mathrm{hPa}$ (triangles in Fig. 2). The region of wave evanescence and EPF divergence extends lower and more poleward. The changes in the vertical reflecting surface and index of refraction are suggestive of a weakened wave flux into the subtropical upper stratosphere. We thus conclude that linear theory, but not critical layer predictions, can explain how shear in the upper stratosphere may affect subtropical Rossby wave propagation.

\section{Discussion and conclusions}

In this paper, a comprehensive GCM (WACCM) and a hierarchy of diagnostics are used to demonstrate that linear theory can describe the changes in Rossby wave propagation in response to easterly QBO winds. In the stratosphere, significant changes occur in three regions: the subtropical lower stratosphere, the subtropical upper stratosphere, and the polar vortex. The changes occur as follows:

(i) The introduction of tropical lower stratospheric easterlies enhances synoptic Rossby wave convergence in the subtropical lower stratosphere, thereby extending poleward the tropical easterlies (and critical line) and warming the subtropical lower stratosphere. The response in this region (denoted "1" in Fig. 1) is consistent with the Holton-Tan mechanism.

(ii) The meridional circulation of the QBO (but not the critical line) influences wave propagation out to $50^{\circ} \mathrm{N}$ in the upper stratosphere (denoted " 2 " in Fig. 1), whereby equatorward planetary Rossby wave propagation from subpolar latitudes into the subtropics is reduced. This change in wave propagation leads to accelerated subtropical upper stratospheric winds and weakened subpolar and polar stratospheric winds. Enhanced EPF convergence in the polar stratosphere initially weakens the polar vortex at upper levels only. After the upper stratospheric vortex begins to weaken, temperature anomalies propagate downward into the middle and lower stratosphere. 
EQBOequil - neutQBO, EPF and divergence perp. Jan
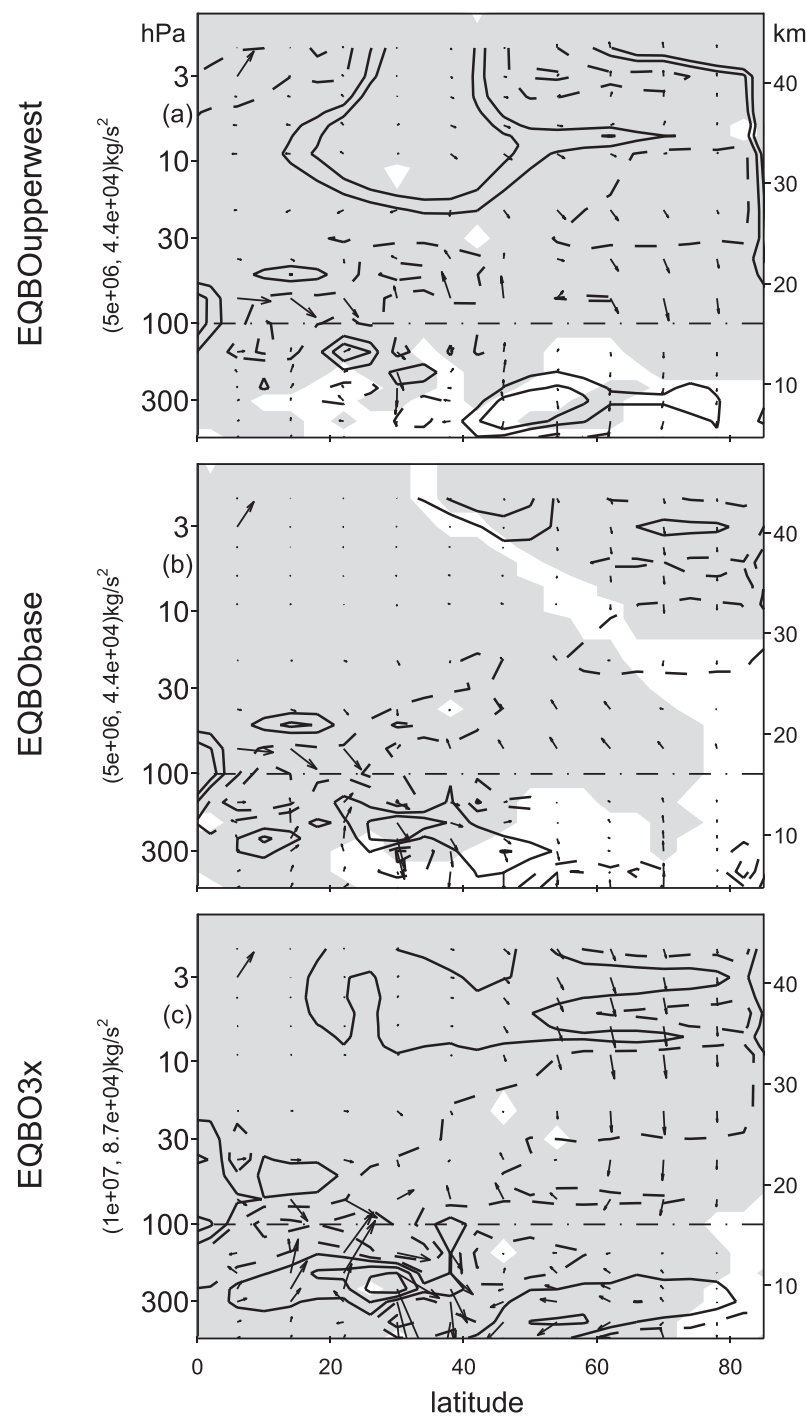

FIG. 14. Difference in EP flux between the perpetual January equilibrium EQBO cases and the neutQBO case for EQBO winds with (a) upper stratospheric westerlies and (b) neutral QBO upper stratospheric winds; (c) as in (b), but with wind anomalies 3 times stronger. Plotting conventions as in the top row of Fig. 8 for (a) and (b) and bottom row of Fig. 8 for (c). The arrow lengths for the $3 \times$ EQBO are double that for the sheared and nonsheared EQBO cases. Negative contours are dashed.

While linear theory can explain all of these effects, the evolution of the entire wind distribution is only quasilinear because induced zonal wind anomalies cause the wave convergence to move, which then positively feeds back on the zonal wind anomalies. Although unrelated variability can quite effectively mask the mechanism discussed, we expect that linear theory explains much of the response in the (real) atmosphere as well.
The WACCM simulations and the QG diagnostics show that the mean meridional circulation associated with lower stratospheric QBO winds is the crucial driver for the effect on the polar vortex. In contrast, the subtropical zero wind line, downward propagation of equatorial QBO anomalies, anomalies in the troposphere, and QBO upper and midstratospheric winds, are comparatively unimportant for anomalies in the polar stratosphere.

We wish to emphasize that our mechanism involving the meridional circulation of the QBO is distinct from that proposed in Holton and Tan (1980). For example, Holton and Tan (1980) "hypothesize that shifts in the latitude of the zero mean wind line (critical line) associated with the equatorial QBO may be responsible for the planetary wave portion of the extratropical $50 \mathrm{mb}$ QBO," and note that "at present we can only speculate that the latitudinal shift of the $\bar{u}=0$ line might be an important link between the equatorial and high-latitude QBOs," but do not mention other mechanisms. In contrast, we find that the mean meridional circulation associated with lower stratospheric QBO winds is more important and that the altered critical line only influences the subtropics and midlatitudes.

The diagnostics examined suggest that the impact of EQBO winds is dependent on the zonal wavenumber of the wave. Changes in the polar vortex region are strongest for zonal wavenumber 1 , while changes in the subtropical lower stratosphere are driven by higher wavenumbers (including transient synoptic-scale waves). Further work is needed to relate the wavenumber dependence presented here to the seasonally varying wavenumber dependence in Naoe and Shibata (2010), Hu and Tung (2002), and Ruzmaikin et al. (2005). It is conceivable that the enhanced synoptic Rossby wave convergence in the subtropical lower stratosphere contributes to the effect of the QBO on midlatitude lower stratospheric ozone. Such a mechanism was not considered by Gray and Pyle (1989), Kinnersley (1999), or Kinnersley and Tung (1999). As our experiments do not have interactive chemistry, additional investigation is left for future work.

Gray et al. (2001, 2004) and Pascoe et al. (2006) found that major SSW frequency and winter vortex evolution is influenced by winds in the equatorial upper stratosphere, in apparent contradiction to our result that upper stratospheric shear is not important for the HT effect. We emphasize that these authors were primarily examining the effect of wind anomalies above $32 \mathrm{~km}$ $(\sim 7 \mathrm{hPa})$, while we are examining the effect of wind shear between 30 and $7 \mathrm{hPa}$. Further analysis is needed before detailed comparisons can be made. We also note that our model has too little stratospheric polar vortex variability and too few major SSWs. We therefore cannot meaningfully analyze the effect of upper stratospheric QBO winds on major SSW frequency. Even though model 
configurations with a more realistic sudden warming frequency are necessary to extend the results shown here, the linear arguments presented are relevant to (and should be present in) all GCMs.

Naoe and Shibata (2010) and Yamashita et al. (2011) found that WQBO leads to weakened wave propagation near $50 \mathrm{hPa}$ and $40^{\circ} \mathrm{N}$, with presumably enhanced wave propagation in this region for EQBO (as we find). Naoe and Shibata (2010) argue that this effect contradicts the HT mechanism. We argue that this effect is consistent with linear theory, although it may only be tangentially related to the effect near the polar vortex and changes in the subtropical critical line. The region just above the jet core, from which waves are refracted away climatologically, shrinks in response to EQBO winds, and expands in response to WQBO winds. It is therefore expected that waves will diverge away from this region in the composites of Naoe and Shibata (2010) and Yamashita et al. (2011), just as they converge into this region in our plots. Linear theory can explain the changes in the subtropical lower stratosphere in response to the QBO.

OY92 found that linear theory appears incapable of describing the polar upper stratospheric response, because polar regions are only influenced by the QBO if a subtropical "surf zone" can form. Their model does not generate the mean meridional circulation of the QBO (as they note themselves), however, and we find that the meridional circulation of the $\mathrm{QBO}$ is vital for changes in midlatitude index of refraction and EPF. Differences between our results and those in OY92 can therefore be explained by the lack of realism in the model used by OY92. Future work is needed to quantify the relative importance of linear theory and the formation of a nonlinear surf zone for the polar response to the QBO in nature.

Previous work has produced mixed results on how the QBO modulates the polar vortex. We find that quasilinear theory explains the changes in Rossby wave propagation and convergence. The lack of sudden stratospheric warmings, interactive chemistry, and a seasonal cycle in our model suggests that these features are relatively unimportant for the mechanism by which the QBO influences the extratropical stratosphere, although future work is certainly needed. Nevertheless, the dynamics underlying a complex physical process, like the effect of the QBO on Rossby wave propagation, can be more readily understood in models where external unrelated variability is not simulated. The results presented here in a simpler modeling framework should enable a more effective and efficient understanding of the dynamics in more comprehensive (yet complicated) models.

Acknowledgments. This work was supported by the National Science Foundation under Grant AGS 0960497 at the University of Washington and by the National Aeronautical and Space Administration under Grant NNX06AE70G at Johns Hopkins University. We thank the developers at NCAR for the WACCM 3.1.9 and Marc L. Michelsen for assisting our model runs. TAS acknowledges support from the Natural Sciences and Engineering Research Council of Canada through a post-doctoral fellowship.

\section{REFERENCES}

Baldwin, M. P., and T. J. Dunkerton, 2001: Stratospheric harbingers of anomalous weather regimes. Science, 294, 581-584.

Bretherton, C. S., M. Widmann, V. P. Dymnikov, J. M. Wallace, and I. Bladé, 1999: The effective number of spatial degrees of freedom of a time-varying field. J. Climate, 12, 1990-2009.

Calvo, N., M. A. Giorgetta, and C. Peña-Ortiz, 2007: Sensitivity of the boreal winter circulation in the middle atmosphere to the quasi-biennial oscillation in MAECHAM5 simulations. J. Geophys. Res., 112, D10124, doi:10.1029/2006JD007844.

$\_,-,$R. Garcia-Herrera, and E. Manzini, 2009: Nonlinearity of the combined warm ENSO and QBO effects on the Northern Hemisphere polar vortex in MAECHAM5 simulations. J. Geophys. Res., 114, D13109, doi:10.1029/2008JD011445.

Charney, J. G., and P. G. Drazin, 1961: Propagation of planetaryscale disturbances from the lower into the upper atmosphere. J. Geophys. Res., 66, 83-109.

Chen, W., and T. Li, 2007: Modulation of northern hemisphere wintertime stationary planetary wave activity: East Asian climate relationships by the quasi-biennial oscillation. J. Geophys. Res., 112, D20120, doi:10.1029/2007JD008611.

Dunkerton, T. J., and D. P. Delisi, 1985: The subtropical mesospheric jet observed by the Nimbus 7 limb infrared monitor of the stratosphere. J. Geophys. Res., 90, 10 681-10 692.

Fletcher, C., and P. Kushner, 2011: The role of linear interference in the annular mode response to tropical SST forcing. J. Climate, 24, 778-794.

Garcia, R. R., D. Marsh, D. Kinnison, B. Boville, and F. Sassi, 2007: Simulations of secular trends in the middle atmosphere, 19502003. J. Geophys. Res., 112, D09301, doi:10.1029/2006JD007485.

Garfinkel, C. I., and D. L. Hartmann, 2007: Effects of the El NiñoSouthern Oscillation and the quasi-biennial oscillation on polar temperatures in the stratosphere. J. Geophys. Res., 112, D19112, doi:10.1029/2007JD008481.

$\longrightarrow$, and - 2008: Different ENSO teleconnections and their effects on the stratospheric polar vortex. J. Geophys. Res., 113, D18114, doi:10.1029/2008JD009920.

— and _ 2010: The influence of the quasi-biennial oscillation on the North Pacific and El Niño teleconnections. J. Geophys. Res., 115, D20116, doi:10.1029/2010JD014181.

- and - 2011a: The influence of the quasi-biennial oscillation on the troposphere in wintertime in a hierarchy of models. Part I: Simplified dry GCMs. J. Atmos. Sci., 68, 12731289.

$\longrightarrow$, and $-2011 \mathrm{~b}$ : The influence of the quasi-biennial oscillation on the troposphere in wintertime in a hierarchy of models. Part II: Perpetual winter WACCM runs. J. Atmos. Sci., 68, 2026-2041.

,-- , and F. Sassi, 2010: Tropospheric precursors of anomalous Northern Hemisphere stratospheric polar vortices. J. Climate, 23, 3282-3299. 
Gray, L. J., and J. A. Pyle, 1989: A two-dimensional model of the quasi-biennial oscillation of ozone. J. Atmos. Sci., 46, 203-220.

— E. F. Drysdale, B. N. Lawrence, and T. J. Dunkerton, 2001: Model studies of the interannual variability of the northernhemisphere stratospheric winter circulation: The role of the quasi-biennial oscillation. Quart. J. Roy. Meteor. Soc., 127, 1413-1432, doi:10.1002/qj.49712757416.

_, S. Crooks, C. Pascoe, S. Sparrow, and M. Palmer, 2004: Solar and QBO influences on the timing of stratospheric sudden warmings. J. Atmos. Sci., 61, 2777-2796.

Hampson, J., and P. Haynes, 2006: Influence of the equatorial QBO on the extratropical stratosphere. J. Atmos. Sci., 63, 936951.

Harnik, N., 2002: The evolution of a stratospheric wave packet. J. Atmos. Sci., 59, 202-217.

— , and R. S. Lindzen, 2001: The effect of reflecting surfaces on the vertical structure and variability of stratospheric planetary waves. J. Atmos. Sci., 58, 2872-2894.

Hauck, C., and V. Wirth, 2001: Diagnosing the impact of stratospheric planetary wave breaking in a linear model. J. Atmos. Sci., 58, 1357-1370.

Hitchman, M. H., and A. S. Huesmann, 2009: Seasonal influence of the quasi-biennial oscillation on stratospheric jets and Rossby wave breaking. J. Atmos. Sci., 66, 935-946.

Holton, J. R., and C. Mass, 1976: Stratospheric vacillation cycles. J. Atmos. Sci., 33, 2218-2225.

- , and H. C. Tan, 1980: The influence of the equatorial quasibiennial oscillation on the global circulation at $50 \mathrm{mb}$. J. Atmos. Sci., 37, 2200-2208.

_- and J. Austin, 1991: The influence of the equatorial QBO on sudden stratospheric warmings. J. Atmos. Sci., 48, 607-618.

Hu, Y., and K. K. Tung, 2002: Tropospheric and equatorial influences on planetary-wave amplitude in the stratosphere. Geophys. Res. Lett., 29, 1019, doi:10.1029/2001GL013762.

Hurwitz, M. M., P. A. Newman, and C. I. Garfinkel, 2011: The Arctic vortex in March 2011: A dynamical perspective. Atmos. Chem. Phys., 11, 11 447-11 453, doi:10.5194/acp-11-11447-2011.

Kinnersley, J. S., 1999: Seasonal asymmetry of the low- and middlelatitude QBO circulation anomaly. J. Atmos. Sci., 56, 11401153.

— , and K. K. Tung, 1999: Mechanisms for the extratropical QBO in circulation and ozone column. J. Atmos. Sci., 56, 1942-1962.

Limpasuvan, V., D. W. J. Thompson, and D. L. Hartmann, 2004: The life cycle of the Northern Hemisphere sudden stratospheric warmings. J. Climate, 17, 2584-2596.

Marsh, D., R. R. Garcia, D. E. Kinnison, B. A. Boville, F. Sassi, S. C. Solomon, and K. Matthes, 2007: Modeling the whole atmosphere response to solar cycle changes in radiative and geomagnetic forcing. J. Geophys. Res., 112, D23306, doi: 10.1029/2006JD008306.

Matsuno, T., 1970: Vertical propagation of stationary planetary waves in the winter Northern Hemisphere. J. Atmos. Sci., 27, 871-883.

Matthes, K., D. R. Marsh, R. R. Garcia, D. E. Kinnison, F. Sassi, and S. Walters, 2010: Role of the QBO in modulating the influence of the 11 year solar cycle on the atmosphere using constant forcings. J. Geophys. Res., 115, D18110, doi:10.1029/ 2009JD013020.

Naito, Y., and S. Yoden, 2006: Behavior of planetary waves before and after stratospheric sudden warming events in several phases of the equatorial QBO. J. Atmos. Sci., 63, 1637-1649.

_- M. Taguchi, and S. Yoden, 2003: A parameter sweep experiment on the effects of the equatorial QBO on stratospheric sudden warming events. J. Atmos. Sci., 60, 1380-1394.

Naoe, H., and K. Shibata, 2010: Equatorial quasi-biennial oscillation influence on northern winter extratropical circulation. J. Geophys. Res., 115, D19102, doi:10.1029/2009JD012952.

Niwano, M., and M. Takahashi, 1998: The influence of the equatorial QBO on the Northern Hemisphere winter circulation of a GCM. J. Meteor. Soc. Japan, 76, 453-461.

O'Sullivan, D., and R. Young, 1992: Modeling the quasi-biennial oscillation's effect on the winter stratospheric circulation. J. Atmos. Sci., 49, 2437-2448.

— extratropical QBO in a numerical model of the middle atmosphere. J. Atmos. Sci., 51, 3706-3721.

Pascoe, C. L., L. J. Gray, and A. A. Scaife, 2006: A GCM study of the influence of equatorial winds on the timing of sudden stratospheric warmings. Geophys. Res. Lett., 33, L06825, doi: 10.1029/2005GL024715.

Perlwitz, J., and N. Harnik, 2003: Observational evidence of a stratospheric influence on the troposphere by planetary wave reflection. J. Climate, 16, 3011-3026.

Plumb, R. A., 1982: Zonally symmetric Hough modes and meridional circulations in the middle atmosphere. J. Atmos. Sci., 39, 983-991.

_ , and R. C. Bell, 1982: A model of the quasi-biennial oscillation on an equatorial beta-plane. Quart. J. Roy. Meteor. Soc., 108, 335-352, doi:10.1256/smsqj.45603.

Randel, W. J., and I. M. Held, 1991: Phase speed spectra of transient eddy fluxes and critical layer absorption. J. Atmos. Sci., 48, 688-697.

Richter, J. H., F. Sassi, R. R. Garcia, K. Matthes, and C. A. Fischer, 2008: Dynamics of the middle atmosphere as simulated by the Whole Atmosphere Community Climate Model, version 3 (WACCM3). J. Geophys. Res., 113, D08101, doi:10.1029/ 2007JD009269.

Ruzmaikin, A., J. Feynman, X. Jiang, and Y. L. Yung, 2005: Extratropical signature of the quasi-biennial oscillation. J. Geophys. Res., 110, D11111, doi:10.1029/2004JD005382.

Shaw, T. A., J. Perlwitz, and N. Harnik, 2010: Downward wave coupling between the stratosphere and troposphere: The importance of meridional wave guiding and comparison with zonal-mean coupling. J. Climate, 23, 6365-6381.

Wei, K., W. Chen, and R. Huang, 2007: Association of tropical Pacific sea surface temperatures with the stratospheric HoltonTan oscillation in the Northern Hemisphere winter. Geophys. Res. Lett., 34, L16814, doi:10.1029/2007GL030478.

Yamashita, Y., H. Akiyoshi, and M. Takahashi, 2011: Dynamical response in the Northern Hemisphere midlatitude and highlatitude winter to the QBO simulated by CCSR/NIES CCM. J. Geophys. Res., 116, D06118, doi:10.1029/2010JD015016. 